\title{
On the gas temperature in circumstellar disks around $A$ stars
}

\author{
I. Kamp and G.-J. van Zadelhoff
}

Leiden Observatory, PO Box 9513, 2300 RA Leiden, The Netherlands

Received 18 December 2000 / Accepted 2 May 2001

\begin{abstract}
In circumstellar disks or shells it is often assumed that gas and dust temperatures are equal where the latter is determined by radiative equilibrium. This paper deals with the question whether this assumption is applicable for tenuous circumstellar disks around young A stars. In this paper the thin hydrostatic equilibrium models described by Kamp \& Bertoldi (2000) are combined with a detailed heating/cooling balance for the gas. The most important heating and cooling processes are heating through infrared pumping, heating due to the drift velocity of dust grains, and fine structure and molecular line cooling. Throughout the whole disk gas and dust are not efficiently coupled by collisions and hence their temperatures are quite different. Most of the gas in the disk models considered here stays well below $300 \mathrm{~K}$. In the temperature range below $300 \mathrm{~K}$ the gas chemistry is not much affected by $T_{\text {gas }}$ and therefore the simplifying approximation $T_{\text {gas }}=T_{\text {dust }}$ can be used for calculating the chemical structure of the disk. Nevertheless the gas temperature is important for the quantitative interpretation of observations, like fine structure and molecular lines.
\end{abstract}

Key words. molecular processes - circumstellar matter - stars: early-type - stars: individual: Vega stars: individual: $\beta$ Pictoris - planetary systems

\section{Introduction}

In the literature the expression "Vega-type" stars is widely used to characterize main-sequence stars of any spectral type which show an infrared excess. In order to deal with a more homogeneous subgroup this paper concentrates on nearby A dwarfs which are surrounded by dust disks including the most prominent and well studied stars $\beta$ Pictoris and Vega.

The literature presents a large variety of models for these stars ranging from simple spherical shells with constant density (Chini et al. 1990) to detailed disk models with power law density distributions and several dust components (Pantin et al. 1997). These models are used to derive constraints on the properties of the dust components like grain sizes, composition and dust mass in these disks.

Several attempts to detect $\mathrm{CO}$ or other molecules at radio wavelength in the disks around A stars failed (Yamashita et al. 1993; Dent et al. 1995; Liseau 1999). On the other hand circumstellar gas is observed in the visible spectra of several A-type dwarfs (e.g. Holweger \& Rentzsch-Holm 1995; Jolly et al. 1998; Welsh et al. 1998; Holweger et al. 1999; Roberge et al. 2000) proving without doubt the existence of atomic and molecular gas ( $\beta$ Pictoris: $\mathrm{CO})$ in their surrounding.

In order to study the nature of the circumstellar surroundings and the ongoing physical processes in more de-

Send offprint requests to: I. Kamp,

e-mail: kamp@strw.leidenuniv.nl tail Kamp \& Bertoldi (2000) developed disk models comprising the two components gas and dust. These models include a realistic treatment of the UV radiation field and the chemistry by the use of a chemical reaction network. As a first approximation gas and dust are assumed to be effectively coupled by collisions and their temperature is derived from the radiation equilibrium of the dust. The basic result of that work is an explanation of the nondetection of $\mathrm{CO}$ radio lines without assuming a general gas depletion in the disk. The recent detection of $\mathrm{H}_{2}$ in the disk around $\beta$ Pictoris (Thi et al. 2001) can be explained in the context of the above described chemical disk models.

This paper aims at a more realistic determination of the gas temperature in the above cited disk models by the use of a detailed heating/cooling balance. The assumption of $T_{\text {gas }}=T_{\text {dust }}$ will be evaluated. The gas temperature is important for the interpretation of observational data via modelling of the line emission from the gas phase, e.g. CO radio lines, [C II] and [O I] fine structure lines, and narrow circumstellar absorption lines on top of the broadened stellar profile like the ones seen in the Ca II K line.

\section{The circumstellar disk model}

The disk model and the chemical network is described in detail by Kamp \& Bertoldi (2000). We restrict ourselves here to a short summary. 
The density distribution in the disk follows from a thin hydrostatic equilibrium model

$n(r, z)=n_{\mathrm{i}}\left(r / R_{\mathrm{i}}\right)^{-2.5} \mathrm{e}^{-z^{2} / 2 h^{2}}$.

Here the dimensionless scaleheight $H \equiv h / r$ is assumed to be 0.15 . The inner radius of the disk $R_{\mathrm{i}}$ is fixed to $40 \mathrm{AU}$, the outer radius $R_{\mathrm{O}}$ to $500 \mathrm{AU}$. The dust temperature follows from radiative equilibrium assuming large spherical black body grains of size $a$

$T_{\text {dust }}=282.5\left(L_{*} / L_{\odot}\right)^{1 / 5}(r / \mathrm{AU})^{-2 / 5}(a / \mu \mathrm{m})^{-1 / 5}$,

with the stellar luminosity in units of the solar one $L_{*} / L_{\odot}$. Such a disk model leads to a surface density which follows a $r^{-1.2}$ power law in reasonable agreement with the "literature exponents" of brightness profiles (Hayashi et al. 1985; Dutrey et al. 1996; Augereau et al. 2001) ranging from -1 to -1.5 . The radiation field $F_{\nu}$ is described by an ATLAS9 photospheric model (Kurucz 1992) for the appropriate stellar parameters.

We derive a stationary solution for the chemistry using a chemical network, which consists of 47 atomic, ionic and molecular species that are related through 260 gasphase chemical and photoreactions. A number of reactions is treated in more detail like $\mathrm{H}_{2}$ and $\mathrm{CO}$ photodissociation, and $\mathrm{C}$ ionisation. The only surface reactions incorporated are $\mathrm{H}_{2}$ formation and freezing out of $\mathrm{CO}$ on cold dust grain surfaces (see Kamp \& Bertoldi 2000 for further details).

\subsection{The gas temperature}

Kamp \& Bertoldi (2000) assumed that gas and dust are effectively coupled by collisions and hence both have equal temperatures, determined by the radiative equilibrium of the dust. This assumption will be critically evaluated by implementing a detailed heating-cooling balance for the gas phase. The particle densities depend on the gas temperature and vice versa. Hence the energy balance of the gas and the chemical network have to be solved simultaneously. The gas temperature is determined by a detailed energy balance $\Gamma=\Lambda$, where $\Gamma$ and $\Lambda$ are the sum of all relevant heating and cooling rates respectively. To find the solution to $\Gamma-\Lambda=0$, we use Ridder's method, a root bracketing algorithm (Press et al. 1997), in a slightly modified way: as starting values for the gas temperature, we use twice and half the value at the previous radial point. Each change of temperature due to the root bracketing is immediately followed by a solution of the chemical network. If the root bracketing algorithm gets stuck, the routine starts to subsequently extend again the bracketing interval. In smooth areas convergence is normally achieved after 5 iterations.

\subsubsection{Heating processes}

In the following we give a short description of the heating processes taken into account for the determination of the gas temperature.
Photoelectric heating. The photoelectric emission from grain surfaces is a major heating source for the gas (Watson 1972; Draine 1978). Dust particles in the circumstellar disks considered here have typical sizes of a few microns; hence the properties of these large grains large compared to the wavelength of UV radiation - are well described by those of bulk material. This approach differs from the one of Draine (1978) in that we deal in this paper with micron sized dust instead of small interstellar dust particles. Two different grain compositions are studied: graphite and silicate.

The work functions of these materials are taken from the experimental work of Feuerbacher \& Fitton (1972), $w=4.7 \mathrm{eV}$ for graphite and $w=8.0 \mathrm{eV}$ for silicate. Analytical fits to their data describe the photoelectric yields $Y$ as functions of the energy of the incoming UV photon $h \nu$

$\log Y(h \nu)=\left\{\begin{aligned}-19.0 \exp (-0.263 h \nu)- & 1.44 \\ & \text { for graphite } \\ -300.0 \exp (-0.577 h \nu)- & 1.06 \\ & \text { for silicate. }\end{aligned}\right.$

The kinetic energy spectrum of the emitted photoelectron is crudely approximated by Eq. (3) of Draine (1978)

$f(E, h \nu)=(h \nu-w)^{-1} \quad$ if $0<E<h \nu-w$.

Here $E$ denotes the kinetic energy of the escaping photoelectron.

The photoelectric heating rate depends on the energy of the impinging UV photon and on the grain potential $U$. The currents that determine the grain potential are impinging electrons and UV photons. The contribution of impinging ions, mainly protons, is negligible, because hydrogen is mostly neutral or even molecular in these disks. The incident electron current is (Draine 1978)

$J_{\mathrm{ec}}= \begin{cases}n_{\mathrm{e}} s_{\mathrm{e}}\left(\frac{k T}{2 \pi m_{\mathrm{e}}}\right)^{\frac{1}{2}}(1+\phi) & \text { if } \phi \geq 0 \\ n_{\mathrm{e}} s_{\mathrm{e}}\left(\frac{k T}{2 \pi m_{\mathrm{e}}}\right)^{\frac{1}{2}} \mathrm{e}^{\phi} & \text { if } \phi<0,\end{cases}$

with $\phi \equiv e U / k T, m_{\mathrm{e}}$ the electron mass and $n_{\mathrm{e}}$ the electron density. The sticking probability of electrons to grains $s_{\mathrm{e}}$ is assumed to be 0.5 following the argumentation of Draine (1978).

Photoelectrons will be emitted from the grains at a rate per grain surface area given by (Draine 1978)

$J_{\mathrm{pe}}=\int_{E_{\min }}^{E_{\max }}\left(\int_{\nu_{\mathrm{th}}}^{\nu_{\max }} Q_{\mathrm{abs}} Y(h \nu) f(E, h \nu) F_{\nu} \mathrm{d} \nu\right) \mathrm{d} E$,

with $E_{\min }=0$ for $U<0, E_{\min }=e U$ for $U \geq 0$ and $E_{\max }=h \nu_{\max }-w$. The absorption efficiency of the dust grains $Q_{\mathrm{abs}}$ is assumed to be 1 independent of wavelength in the UV. The frequency $\nu_{\text {th }}$ denotes that of impinging UV photons at the threshold $w$. The equilibrium grain potential $U$ can be derived from Eqs. (5) and (6). 


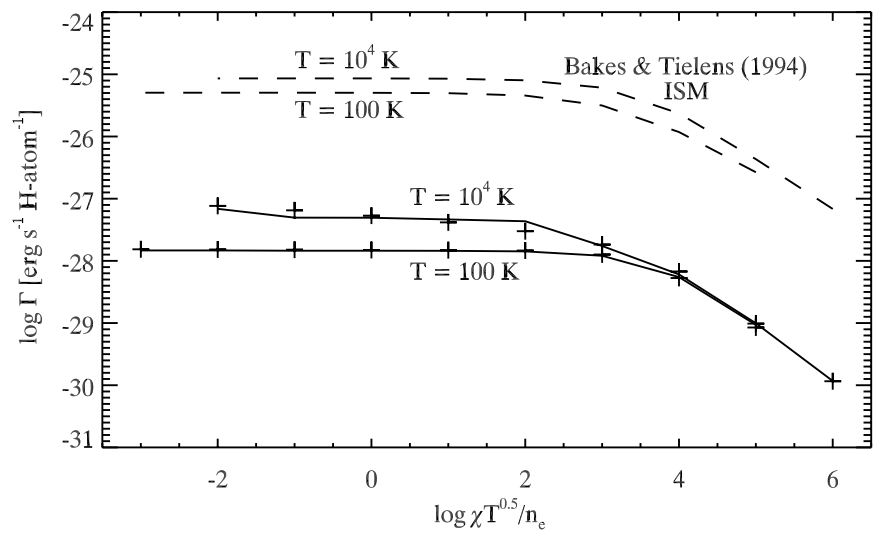

Fig. 1. A comparison between the exact photoelectric heating rate (plus signs), the analytical approximation for large graphite grains (Eq. (8): solid line, $\sigma=2.34 \times$ $10^{-23} \mathrm{~cm}^{2} \mathrm{H}$-atom ${ }^{-1}$ ) and the analytical approximation for small grains (Bakes \& Tielens 1994: dashed line) as a function of the grain charge parameter assuming ISM conditions, $\chi=1$, and two different gas temperatures $T=100 \mathrm{~K}$ and $10^{4} \mathrm{~K}$.

Given the grain potential and the stellar UV radiation field, the photoelectric heating rate can be determined from

$$
\begin{aligned}
& \Gamma_{1}=4 n_{\mathrm{H}} \sigma \int_{E_{\min }}^{E_{\max }}\left(\int_{\nu_{\mathrm{th}}}^{\nu_{\max }} Q_{\mathrm{abs}} Y(h \nu) f(E, h \nu) F_{\nu} \mathrm{d} \nu\right) \\
& (E-e U) \mathrm{d} E \quad \operatorname{erg} \mathrm{cm}^{-3} \mathrm{~s}^{-1} \text {, }
\end{aligned}
$$

where $n_{\mathrm{H}}$ and $\sigma$ denote the particle density of hydrogen and the grain geometric cross section per $\mathrm{H}$ nucleus.

In order to make the calculation of the photoelectric heating rate efficient enough for the inclusion into the heating/cooling balance, the rate is approximated using a function similar to Bakes \& Tielens (1994)

$\Gamma_{\mathrm{pe}}=10^{-4} \sigma \epsilon \chi n_{\mathrm{H}}$

for electron particle densities $10^{-5}<n_{\mathrm{e}}<10^{5} \mathrm{~cm}^{-3}$, gas temperatures $10<T<10000 \mathrm{~K}$, and FUV photon fluxes $10^{-5}<\chi<10^{5}$, where the flux is measured in units of the Habing (1968) field between $912-1110 \AA\left(F_{\mathrm{H}}=\right.$ $\left.1.2 \times 10^{7} \mathrm{~cm}^{-2} \mathrm{~s}^{-1}\right)$.

The photoelectric efficiency $\epsilon$ for graphite is approximated by

$\epsilon=\frac{6 \times 10^{-2}}{1+2.5 \times 10^{-4} x^{0.95}}+\frac{y\left(10^{-4} T\right)}{1+10^{-2} x}$,

where $y$ depends on the so-called grain charge parameter $x \equiv \chi T^{0.5} / n_{\mathrm{e}}$

$y= \begin{cases}4.3 \times 10^{-1} & \text { if } x \leq 10^{-4} \\ 2.3 \times 10^{-1} & \text { if } \quad 10^{-4}<x \leq 10^{-1} \\ 1.5 \times 10^{-1} & \text { if } 10^{-1}<x \leq 10^{2} \\ 2.5 \times 10^{-1} & \text { if } x>10^{2} .\end{cases}$

This formula yields photoelectric heating rates accurate to within 10-25\%.

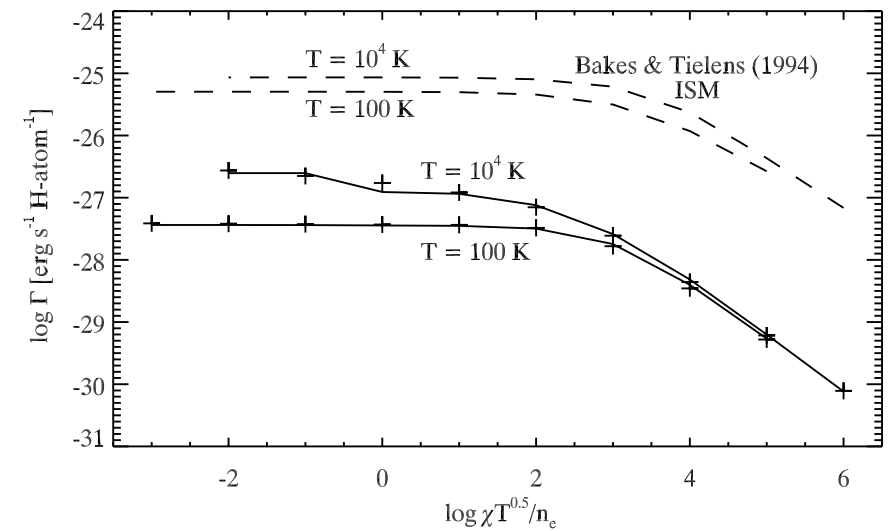

Fig. 2. Same as Fig. 1 but for large silicate grains.

The photoelectric heating rate for silicate grains is slightly different from the one for graphite and reads as follows

$\Gamma_{\mathrm{pe}}=2.5 \times 10^{-4} \sigma \epsilon \chi n_{\mathrm{H}}$

with

$\epsilon=\frac{6 \times 10^{-2}}{1+1.8 \times 10^{-3} x^{0.91}}+\frac{y\left(10^{-4} T\right)^{1.2}}{1+10^{-2} x}$,

and

$y=\left\{\begin{array}{lll}7 \times 10^{-1} & \text { if } \quad x \leq 10^{-4} \\ 3.6 \times 10^{-1} & \text { if } \quad 10^{-4}<x \leq 1 \\ 1.5 \times 10^{-1} & \text { if } x>1\end{array}\right.$

Figure 1 compares the photoelectric heating rate derived in this paper for large graphite grains with that derived by Bakes \& Tielens (1994) for ISM graphite

$\Gamma_{\mathrm{pe}}=10^{-24} \epsilon \chi n_{\mathrm{H}}$,

where the photoelectric efficiency $\epsilon$ is approximated by

$\epsilon=\frac{0.0487}{1+4 \times 10^{-3} x^{0.73}}+\frac{0.0365\left(10^{-4} T\right)^{0.7}}{1+2 \times 10^{-4} x}$.

The difference is due to the larger photoelectric yield of small grains. Photoemission is mostly a "volume process". In large grains the escape probability of photoelectrons is reduced, because they are created far below the surface. There scattering becomes important and they can easily be retrapped in the grain. Figure 2 reveals the same effect for the large silicate grains.

Heating by collisional de-excitation of $\mathrm{H}_{2} . \mathrm{H}_{2}$ molecules excited by Lyman and Werner band absorption decay to vibrotationally excited levels of the ground electronic state and heat the gas via collisional de-excitation. We approximate the complex process using the formula of Tielens \& Hollenbach (1985) for a single excited pseudovibrational level

$$
\begin{aligned}
& \Gamma_{2}=f_{\mathrm{H}_{2}^{*}} n\left(\mathrm{H}_{2}\right) E_{*}\left(1.67 \times 10^{-11} n(\mathrm{H}) \sqrt{T} \mathrm{e}^{-1000 / T}\right. \\
& \left.+2.33 \times 10^{-11} n\left(\mathrm{H}_{2}\right) \sqrt{T} \mathrm{e}^{-18100 /(T+1200)}\right) \\
& \text { erg } \mathrm{cm}^{-3} \mathrm{~s}^{-1} \text {. }
\end{aligned}
$$


$f_{\mathrm{H}_{2}^{*}}$ denotes the fixed fraction of vibrationally excited molecular hydrogen and $E_{*}=4.166 \times 10^{-12} \mathrm{erg}$ is the effective energy of the pseudolevel. We assume in this paper $f_{\mathrm{H}_{2}^{*}}=10^{-5}$.

Heating by photodissociation of $\mathrm{H}_{2}$. Photodissociation of $\mathrm{H}_{2}$ occurs via line transitions to excited levels followed by spontaneous radiative dissociation into two hydrogen atoms. The kinetic energy of each H-atom is typically $0.4 \mathrm{eV}$ (Stephens \& Dalgarno 1973), leading to an approximate heating rate

$\Gamma_{3}=6.4 \times 10^{-13} \Gamma_{\mathrm{H}_{2}} n\left(\mathrm{H}_{2}\right) \quad$ erg $\mathrm{cm}^{-3} \mathrm{~s}^{-1}$.

$\Gamma_{\mathrm{H}_{2}}=\chi \zeta_{0} f_{\text {shield }}^{\mathrm{H}_{2}} \mathrm{~s}^{-1}$ denotes the $\mathrm{H}_{2}$ photodissociation rate, which depends on the strength of the UV radiation field and on the amount of $\mathrm{H}_{2}$ self-shielding (Kamp \& Bertoldi 2000).

Heating by $\mathrm{H}_{2}$ formation on dust. The formation of an $\mathrm{H}_{2}$ molecule on the surface of a dust grain releases the binding energy of $4.48 \mathrm{eV}$. Due to the lack of laboratory data, we follow the approach of Black \& Dalgarno (1976) and assume that this energy goes into translation, vibration and rotation in equal parts. Hence about $1.5 \mathrm{eV}$ will go into kinetic energy and therefore heat the gas

$\Gamma_{4}=2.39 \times 10^{-12} R_{\text {form }} n(\mathrm{H}) n_{\text {tot }} \quad \operatorname{erg~cm}^{-3} \mathrm{~s}^{-1}$.

$R_{\text {form }}$ is the temperature dependent $\mathrm{H}_{2}$ formation rate (Kamp \& Bertoldi 2000).

Gas-grain collisions. Gas-grain collisions will act as a gas heating source for dust temperatures larger than the gas temperature (Burke \& Hollenbach 1983),

$$
\begin{aligned}
\Gamma_{5}= & 4.0 \times 10^{-12} \pi a^{2} n_{\text {tot }} n_{\text {dust }} \alpha_{\mathrm{T}} \sqrt{T}\left(T_{\text {dust }}-T\right) \\
& \operatorname{erg~cm}{ }^{-3} \mathrm{~s}^{-1}
\end{aligned}
$$

where $n_{\text {dust }}$ and $\alpha_{\mathrm{T}}$ denote the particle density of dust grains and the thermal accomodation coefficient respectively. A typical value for silicate and graphite dust is $\alpha_{\mathrm{T}}=0.3$ (Burke \& Hollenbach 1983). If the gas temperature exceeds that of the dust, this rate becomes negative and therefore acts as a cooling rate $\left(\Lambda_{5}\right)$.

Heating due to $\mathrm{C}$ ionisation. In the presence of strong UV radiation neutral carbon can be photoionized with an average yield of approximately $1 \mathrm{eV}$ per photoelectron (Black 1987)

$$
\begin{aligned}
& \Gamma_{6}=1.6022 \times 10^{-12} \Gamma_{\mathrm{C}} n(\mathrm{C}) f_{\text {shield }}(\mathrm{C}) \chi_{\mathrm{x}} f_{\text {shield }}\left(\mathrm{H}_{2}\right) \\
& \operatorname{erg~} \mathrm{cm}^{-3} \mathrm{~s}^{-1} \text {, }
\end{aligned}
$$

where $\chi_{\mathrm{x}}$ is the strength of the UV radiation field attenuated by dust. The carbon self-shielding factor

$$
f_{\text {shield }}(\mathrm{C})=\exp \left(-N(\mathrm{C}) a_{\mathrm{C}}\right)
$$

depends on the $\mathrm{C}$ column density $N(\mathrm{C})$ and on the average carbon ionization cross section $a_{\mathrm{C}}$. The attenuation by $\mathrm{H}_{2}$ can be approximated (Kamp \& Bertoldi 2000) by

$$
f_{\text {shield }}\left(\mathrm{H}_{2}\right)=\exp \left(-T_{\text {gas }}^{0.25}\left(N\left(\mathrm{H}_{2}\right) / 10^{22}\right)^{0.45}\right) \text {. }
$$

Drift velocity of the dust grains. Assuming typical physical conditions for these circumstellar disks, gas and dust are momentum coupled (Gilman 1972) and we assume that all the momentum gained by the grains from the radiation field is transferred to the gas by collisions. Dust grains of micron-size are usually removed by radiation pressure from the disk on timescales short compared to the lifetime of the disk (Artymowicz \& Clampin 1997; van der Bliek et al. 1994; Backman \& Paresce 1993; Artymowicz 1988). Since observations nevertheless reveal the presence of micron-sized dust grains, there must be a process of continuous replenishment for these small grains, for example destructive collisions between much larger dust particles.

Gas and dust in the disk rotate with slightly subkeplerian speeds $v_{\phi}$ due to the gas pressure (gas component) and due to the drag force between dust and gas (dust component). Hence stellar gravity is largely balanced by centrifugal forces. The evaluation of the drift velocities in both directions leads to a coupled system of two-fluid twodimensional hydrodynamical equations and is beyond the scope of this paper. Hence we restrict ourselves here to the case of maximum drift velocity, that is assuming keplerian rotation, and bracket the "true" solution by $v_{\text {drift }}=0$ and $v_{\text {drift }}=v_{\text {drift }}^{\max }$. The maximum drift velocity can then be derived from the balance of radiation pressure on the grains and drag force due to the grains motion through the gas (Tielens 1983)

$v_{\text {drift }}^{\max }=\left[\frac{1}{2}\left(\left(\left(f_{\text {rad }}\right)^{2}+v_{\text {gas }}^{4}\right)^{0.5}-v_{\text {gas }}^{2}\right)\right]^{0.5} \mathrm{~cm} \mathrm{~s}^{-1}$

with the thermal velocity of the gas

$v_{\text {gas }}=\sqrt{\frac{5 k T}{3 \mu m_{\mathrm{H}}}} \mathrm{cm} \mathrm{s}^{-1}$

and

$f_{\mathrm{rad}}=\frac{L_{*} \bar{Q}_{\mathrm{ext}}}{2 \pi c r^{2} \rho_{\mathrm{tot}}} \mathrm{cm}^{2} \mathrm{~s}^{-2}$.

Here $\sigma_{\mathrm{g}}$ and $m_{\mathrm{g}}$ are the geometrical cross section and the mass of the dust grains, $L_{*}$ and $M_{*}$ are the luminosity and mass of the central star, $\rho_{\text {tot }}$ is the total mass density of the gas, and $\bar{Q}_{\text {ext }}$ is the flux-weighted mean of the radiation pressure efficiency

$\bar{Q}_{\text {ext }}=\frac{\int_{0}^{\infty} Q_{\text {ext }}(\nu) F(\nu) \mathrm{d} \nu}{\int_{0}^{\infty} F(\nu) \mathrm{d} \nu}$

The rate of viscous gas heating is approximated (Tielens 1983) by

$\Gamma_{7}=0.5 \rho_{\text {tot }} n_{\text {dust }} \sigma_{\mathrm{g}} v_{\text {drift }}^{3} \quad \operatorname{erg~} \mathrm{cm}^{-3} \mathrm{~s}^{-1}$. 
Cosmic ray heating. Following Clavel et al. (1978) The cosmic ray heating rate for a mixture of $\mathrm{H}, \mathrm{H}_{2}$ and $\mathrm{He}$ is

$\Gamma_{8}=\left(1+x_{\mathrm{He}}\right) \zeta(\mathrm{H}) n(\mathrm{H})\left(1.28 \times 10^{-11}+2.44 \times 10^{-11} x_{\mathrm{H}_{2}}\right)$ erg $\mathrm{cm}^{-3} \mathrm{~s}^{-1}$,

where $\zeta(\mathrm{H})=6 \times 10^{-18} \mathrm{~s}^{-1}$ is the primary cosmic ray ionisation rate of hydrogen and $x_{\mathrm{He}}$ and $x_{\mathrm{H}_{2}}$ are the abundances of $\mathrm{He}$ and molecular hydrogen relative to atomic hydrogen.

\subsubsection{Cooling processes}

In the following we give a short description of the cooling processes taken into account for the determination of the gas temperature.

O I cooling. At densities larger than $n_{\mathrm{cr}}^{\mathrm{H}}=8.5 \times$ $10^{5}(T / 100)^{-0.69} \mathrm{~cm}^{-3}$, LTE is a good approximation for the oxygen level occupation numbers. However the densities in the outer parts of these disks will be below that critical value. Therefore we have to calculate the statistical equilibrium (SE) of oxygen in detail. More importantly thermal emission of $50 \mathrm{~K}$ dust peaks at $60 \mu \mathrm{m}$, where we also find the [O I] $63.2 \mu \mathrm{m}$ fine-structure line. Since typical dust temperatures in our disks range from 20 to $150 \mathrm{~K}$, this can lead to a strong pumping of the fine-structure levels of neutral oxygen. An additional component to the infrared radiation field $P_{\nu}$ is the cosmic microwave background, which is more important for low rotational levels of molecules like CO.

We use an oxygen model atom consisting of the lowest three fine-structure levels and we take into account the three fine-structure lines at $63.2,145.6$, and $44.0 \mu \mathrm{m}$, and collisions with $\mathrm{H}_{2}, \mathrm{H}$, and electrons (Jaquet et al. 1992; Launay \& Roueff 1977; Bell et al. 1998). We include spontaneous emission as well as absorption and stimulated emission due to the IR radiation field. The importance of the latter two processes for the statistical equilibrium calculations is stressed by Kamp \& van Zadelhoff (2000). Table 1 gives a short overview of the atomic data. The last column gives fits to the critical densities above which collisional de-excitation becomes important

$n_{\mathrm{cr}}=\frac{A_{u l}}{\sum_{i} K_{u i}} \quad \mathrm{~cm}^{-3}$,

where $K_{u i}=\epsilon_{\mathrm{H}} C_{u i}^{\mathrm{H}}+\epsilon_{\mathrm{H}_{2}} C_{u i}^{\mathrm{H}_{2}}+\epsilon_{\mathrm{e}^{-}} C_{u i}^{\mathrm{e}^{-}}$denotes the sum of all collisional transitions from level $u$ to all other levels $i$. The critical densities tabulated refer to a neutral molecular gas with $\epsilon_{\mathrm{H}}=10^{-3}, \epsilon_{\mathrm{H}_{2}}=1.0$, and $\epsilon_{\mathrm{e}^{-}}=10^{-6}$.

The total cooling rate is the sum over all three finestructure transitions

$$
\begin{aligned}
\Lambda_{1}=\sum_{k} h \nu_{u l}[ & n_{u}(\mathrm{O})\left(A_{u l}+B_{u l} P_{\nu}\right) \\
& \left.-n_{l}(\mathrm{O}) B_{l u} P_{\nu}\right] \quad \operatorname{erg~cm}^{-3} \mathrm{~s}^{-1},
\end{aligned}
$$
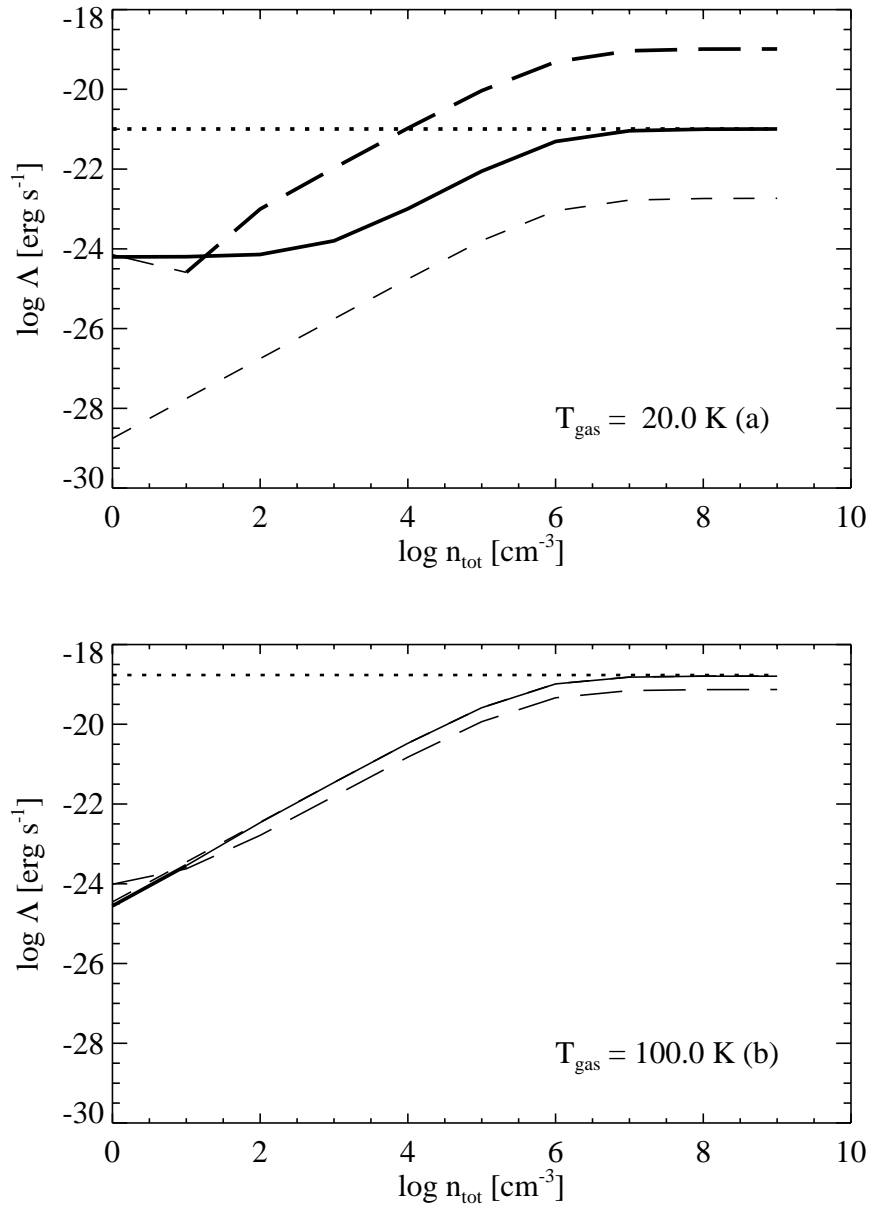

Fig. 3. [O I] fine structure cooling rates for a) $T_{\text {gas }}=20 \mathrm{~K}$ and b) $T_{\text {gas }}=100 \mathrm{~K}$; solid line: $\mathrm{SE}$ including an IR radiation field $P_{\nu}=0.01 B_{\nu}(80 \mathrm{~K})+B_{\nu}(2.7 \mathrm{~K})$, long dashed line: SE including an IR radiation field $P_{\nu}=B_{\nu}(80 \mathrm{~K})+B_{\nu}(2.7 \mathrm{~K})$, dashed line: SE with $P_{\nu}=B_{\nu}(2.7 \mathrm{~K})$, dotted line: LTE including $P_{\nu}=0.01 B_{\nu}(80 \mathrm{~K})+B_{\nu}(2.7 \mathrm{~K})$; thick lines indicate a negative cooling rate (net heating of the gas).

where $A_{u l}$ is the Einstein probability for spontaneous emission, $B_{u l}=c^{2} /\left(2 h \nu_{u l}^{3}\right) A_{u l}$ is the Einstein probability for stimulated emission, and $B_{l u}=g_{u} / g_{l} B_{u l}$ the Einstein coefficient for absorption. The level population numbers $n_{l}(\mathrm{O})$ and $n_{u}(\mathrm{O})$ refer to the lower and upper fine-structure level respectively, and $h \nu_{u l}$ is the energy absorbed from the total infrared radiation field $P_{\nu}$ or radiated away per emission. $\Lambda_{1}$ turns into a heating rate as soon as is becomes negative.

Figure 3 illustrates as an example the influence of an $80 \mathrm{~K}$ IR radiation field on the cooling rate. Assuming black body dust emission, $\Lambda_{1}$ is a net heating rate at low temperatures. Using a more "realistic" radiation field of $0.01 B_{\nu}(80 \mathrm{~K})$, which includes the dilution of radiation due to the low densities in the disk, the heating rate approaches the statistical equilibrium value at particle densities of $10^{7} \mathrm{~cm}^{-3}$. For higher gas temperatures $\left(T_{\text {gas }}>T_{\text {dust }}\right)$ the dust infrared emission is no longer able to heat the gas and LTE is a good approximation for particle densities larger than $10^{7} \mathrm{~cm}^{-3}$. Spontaneous emission 

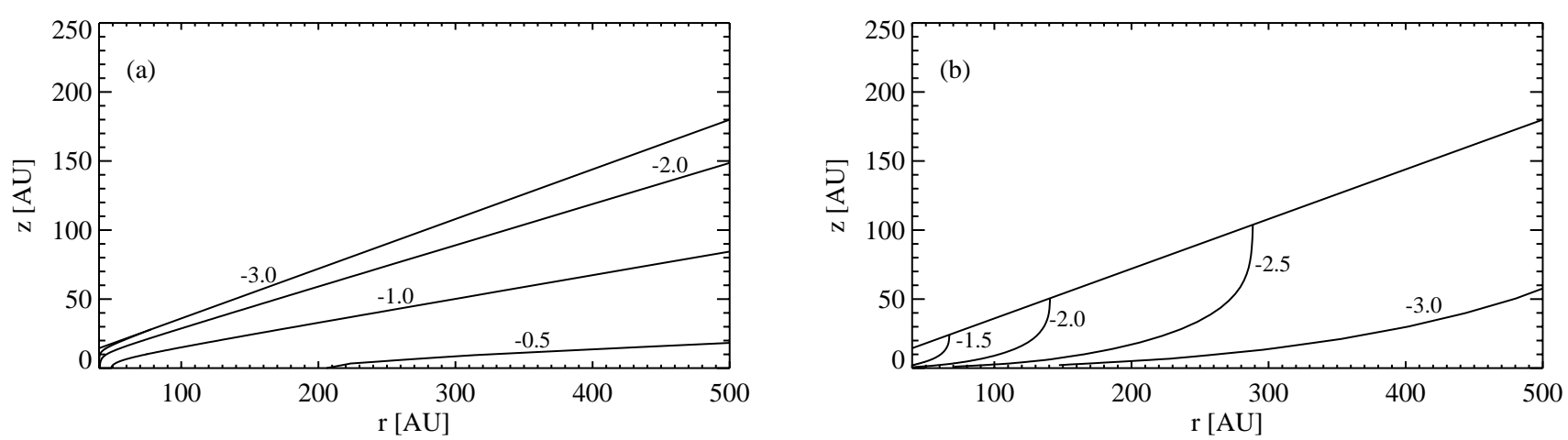

Fig. 4. Optical depth $\log \tau_{63}$ of the [O I] $63.2 \mu \mathrm{m}$ line in a $2 M_{\oplus} \beta$ Pictoris disk model: a) optical depth $\log \tau$ for a photon emitted from the stellar surface, b) optical depth $\log \tau$ for a photon emitted from the disk-midplane.

Table 1. Line data for the oxygen model atom: lower and upper levels of fine-structure line, statistical weights, wavelengths, transition probabilities for spontaneous emission, and fits to the critical densities for the temperature range $10 \mathrm{~K}<T_{\text {gas }}<$ $10^{3} \mathrm{~K}$.

\begin{tabular}{cccccccc}
\hline$u$ & $l$ & $g_{u}$ & $g_{l}$ & $\operatorname{desig}$ & $\lambda[\mu \mathrm{m}]$ & $A_{u l}\left[\mathrm{~s}^{-1}\right]$ & $n_{\text {cr }}\left[\mathrm{cm}^{-3}\right]$ \\
\hline 1 & 0 & 3 & 5 & ${ }^{3} \mathrm{P}_{1}-{ }^{3} \mathrm{P}_{2}$ & 63.2 & $8.87 \times 10^{-5}$ & $3.0 \times 10^{6} T^{-0.35}$ \\
2 & 1 & 1 & 3 & ${ }^{3} \mathrm{P}_{0}-{ }^{3} \mathrm{P}_{1}$ & 145.5 & $1.77 \times 10^{-5}$ & $3.7 \times 10^{5} T^{-0.35}$ \\
2 & 0 & 1 & 5 & ${ }^{3} \mathrm{P}_{0}-{ }^{3} \mathrm{P}_{2}$ & 44.1 & $1.28 \times 10^{-10}$ & $2.7 T^{-0.35}$ \\
\hline
\end{tabular}

dominates the energy balance of $\mathrm{O}$ I and hence the resulting cooling rate is only marginally dependent on the background radiation field.

The optical depth of the strongest [O I] line at $63.2 \mu \mathrm{m}$ can be calculated assuming that all oxygen is in the ground level, and using a typical line width of $\delta v_{\mathrm{d}}=1 \mathrm{~km} \mathrm{~s}^{-1}$

$\tau_{10}=A_{10} \frac{\lambda_{10}^{3}}{8 \pi^{1.5} \delta v_{\mathrm{d}}} \frac{g_{1}}{g_{0}} N_{0}(\mathrm{O})$.

An optical depth $\tau_{10}=1$ is reached for column densities of $N_{0}(\mathrm{O})=3.3 \times 10^{17} \mathrm{~cm}^{-2}$. Assuming that all oxygen is in atomic form and is neutral throughout the whole disk $\left(\log \epsilon_{\mathrm{O}}=-3.49\right)$, this converts into a total column density of $N_{\text {tot }}=1.0 \times 10^{21} \mathrm{~cm}^{-2}$. Using Eq. (4) from Kamp \& Bertoldi (2000), disks up to a total mass of $2 M_{\oplus}$ are optically thin for [O I] $63.2 \mu \mathrm{m}$ radiation. Figure 4 illustrates the optical depth of this line in a $2 M_{\oplus} \beta$ Pictoris disk model for photons emitted from the stellar surface (left) and from the disk-midplane (right).

C II cooling. We take the two level approximation of Hollenbach \& McKee (1989) for the ground state of single ionized carbon; the energies and statistical weights are $E_{0}=0.00 \mathrm{erg}, E_{1}=1.27 \times 10^{-14} \mathrm{erg}$ and $g_{0}=2, g_{1}=4$ respectively. The $157.7 \mu \mathrm{m}$ line, corresponding to a transition from the first excited level $2 \mathrm{P}_{3 / 2}$ to the ground level $2 \mathrm{P}_{1 / 2}$, has a cooling rate of

$\Lambda_{2}=f_{1} n(\mathrm{C}$ II $) A_{10} h \nu_{10} \quad \operatorname{erg~cm}^{-3} \mathrm{~s}^{-1}$, where the fraction of $\mathrm{CI}$ in the upper level is calculated under the assumption of LTE

$f_{1}=\frac{g_{1} \mathrm{e}^{-E_{1} / k T}}{g_{0}+g_{1} \mathrm{e}^{-E_{1} / k T}}$.

The critical density for LTE $n_{\mathrm{cr}}^{\mathrm{H}}=3.0 \times$ $10^{2}(T / 100)^{-0.07} \mathrm{~cm}^{-3}$ is always lower than the minimum density of $10^{4} \mathrm{~cm}^{-3}$ in our disk models. The Einstein probability for spontaneous emission $A_{10}$ is $2.4 \times 10^{-6} \mathrm{~s}^{-1}$

$\mathrm{H}_{2}$ rotational/vibrational line cooling. We use the $\mathrm{H}_{2}$ cooling function derived by Le Bourlot et al. (1999), which can be applied for gas temperatures between 100 and $10^{4} \mathrm{~K}$ and gas densities ranging from 1 to $10^{8} \mathrm{~cm}^{-3}$. Due to the wide energy spacing of the levels, rotational excitation of $\mathrm{H}_{2}$ is negligible below $100 \mathrm{~K}$. Le Bourlot, Pineau des Forêts \& Flower take into account 51 rovibrational energy levels and their collisional excitation by $\mathrm{H}, \mathrm{He}$ and $\mathrm{H}_{2}$ using recent quantum mechanical calculations of the cross sections. Following their results the cooling depends only weakly on the ortho-to-para ratio. We take $n($ ortho $) / n$ (para) $=1.6$, the equilibrium value at $100 \mathrm{~K}$, to be representative for the low gas temperatures in our models (see their Fig. 9).

O I $(6300 \mu \mathrm{m})$ cooling. Line emission from the metastable ${ }^{1} \mathrm{D}$ level of neutral oxygen efficiently cools the gas at very high temperatures. We take the cooling rate from Sternberg \& Dalgarno (1989)

$\Lambda_{4}=1.8 \times 10^{-24} n(\mathrm{O}) n_{\mathrm{e}} \mathrm{e}^{-22800 / T} \quad \operatorname{erg~cm}^{-3} \mathrm{~s}^{-1}$,

where $n(\mathrm{O})$ and $n_{\mathrm{e}}$ denote the density of neutral oxygen and electrons respectively.

Ly $\alpha$ cooling. The cooling by Ly $\alpha$ emission becomes efficient at very high temperatures (Sternberg \& Dalgarno 1989)

$\Lambda_{6}=7.3 \times 10^{-19} n_{\mathrm{e}} n(\mathrm{H}) \mathrm{e}^{-118400 / T} \quad \operatorname{erg~cm}^{-3} \mathrm{~s}^{-1} .(35)$

$n(\mathrm{H})$ and $n_{\mathrm{e}}$ denote the density of neutral hydrogen and electrons respectively. 
$\mathrm{CH}$ cooling. Rotational cooling by $\mathrm{CH}$ is calculated using the optically thin approximation of Hollenbach \& McKee (1979)

$$
L_{\mathrm{rot}}=\left\{\begin{array}{l}
\frac{4(k T)^{2} A_{0}}{n_{\mathrm{tot}} E_{0}\left(1+\left(n_{\mathrm{cr}} / n_{\mathrm{tot}}\right)+1.5\left(n_{\mathrm{cr}} / n_{\mathrm{tot}}\right)^{0.5}\right)} \\
\operatorname{erg~s}^{-1} \mathrm{~cm}^{3} \quad \text { if } n_{\mathrm{tot}} \gg n_{\mathrm{cr}} \\
\frac{k T\left(1-n\left(\mathrm{H}_{2}\right) / n_{\mathrm{tot}}\right) \sigma_{\mathrm{tot}} v_{\mathrm{T}}}{1+\left(n_{\mathrm{tot}} / n_{\mathrm{cr}}\right)+1.5\left(n_{\mathrm{tot}} / n_{\mathrm{cr}}\right)^{0.5}} \\
\mathrm{erg} \mathrm{s}^{-1} \mathrm{~cm}^{3} \quad \text { if } n_{\mathrm{tot}} \ll n_{\mathrm{cr}}
\end{array}\right.
$$

where $n_{\mathrm{cr}}, A_{0}$ and $E_{0}$ are $6.6 \times 10^{9} T_{3}^{-1 / 2} \mathrm{~cm}^{-3}, 7.7 \times$ $10^{-3} \mathrm{~s}^{-1}$ and $2.76 \times 10^{-15}$ erg respectively for the $\mathrm{CH}$ radical. $v_{\mathrm{T}}$ is the thermal velocity of colliding hydrogen atoms. The cooling rate is then

$\Lambda_{7}=n_{\text {tot }} n_{\mathrm{CH}} L_{\text {rot }} \quad \operatorname{erg} \mathrm{cm}^{-3} \mathrm{~s}^{-1}$,

where $n_{\mathrm{CH}}$ denotes the particle density of the $\mathrm{CH}$ molecule.

CO cooling For the $\mathrm{CO}$ molecule the occupation numbers of the rotational lines in the ground-vibrational state differ significantly from LTE for densities below $10^{7} \mathrm{~cm}^{-3}$. Therefore we use a $\mathrm{CO}$ model molecule to calculate the statistical equilibrium of carbon monoxide. It consists of 26 levels up to $J=25$ and has $351 \mathrm{CO}-\mathrm{H}_{2}$ collisional rate coefficients (Schinke et al. 1985). The pumping of CO levels by IR radiation is included in the same way as for O I (see respective paragraph). The molecular line data is summarized in Table 2. The last column of this table gives the best fits to the critical densities for the CO rotational lines using the same assumptions as made for the critical densities of $\mathrm{O}$ I.

$\mathrm{CO}$ rotational cooling is calculated in the optically thin limit by summing over all 25 rotational line transitions

$$
\begin{aligned}
\Lambda_{8}=\sum_{i=1}^{25} h \nu_{i j}[ & n_{i}(\mathrm{CO})\left(A_{i j}+B_{i j} P_{\nu}\right) \\
& \left.\quad n_{j}(\mathrm{CO}) B_{j i} P_{\nu}\right] \operatorname{erg~cm}{ }^{-3} \mathrm{~s}^{-1},
\end{aligned}
$$

with $j=i-1$. $n_{i}(\mathrm{CO})$ denotes the occupation number for the level $J$ and $A_{i j}$ is the spontaneous emission probability for the rotational transition $J \rightarrow J-1 . B_{i j}$ and $B_{j i}$ are the Einstein coefficients for stimulated emission and absorption respectively and $h \nu_{i j}$ is the released or absorbed energy. For photons travelling along the disk midplane, some of the CO lines will become optically thick. Nevertheless this does not influence the cooling rate, as the energy is radiated isotropically in all directions and most of it can therefore escape the flat disk without any reabsorption.

McKee et al. (1982) determined numerical fits for the CO cooling rate for gas temperature larger than $150 \mathrm{~K}$. These cooling rates are derived under the following assumptions: 1 . optically thin lines, 2 . infrared pumping by
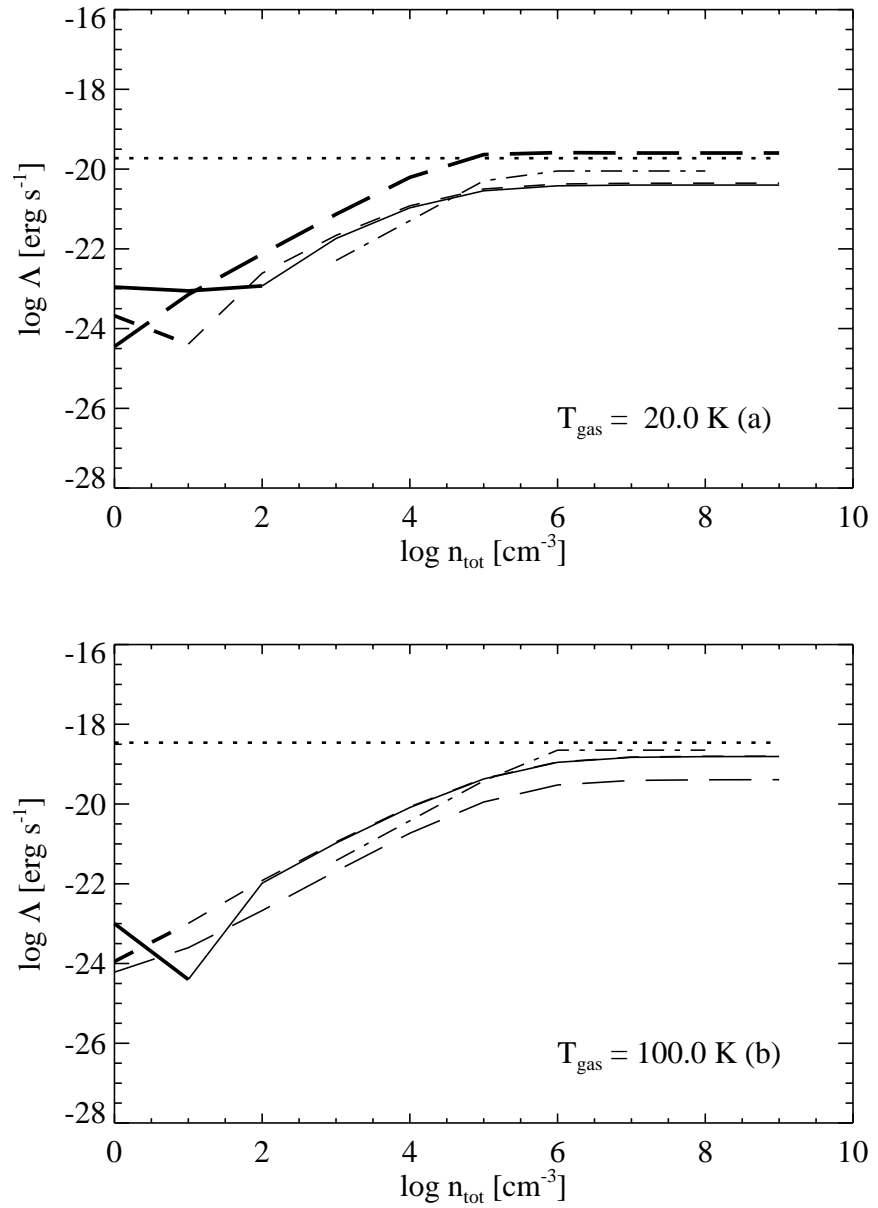

Fig. 5. CO rotational line cooling rates for a) $T_{\text {gas }}=20 \mathrm{~K}$ and $\mathbf{b}) T_{\text {gas }}=100 \mathrm{~K}$; solid line: SE including an IR radiation field $P_{\nu}=0.01 B_{\nu}(80 \mathrm{~K})+B_{\nu}(2.7 \mathrm{~K})$, long dashed line: SE including an IR radiation field $P_{\nu}=B_{\nu}(80 \mathrm{~K})+B_{\nu}(2.7 \mathrm{~K})$, dashed line: SE with $P_{\nu}=B_{\nu}(2.7 \mathrm{~K})$, dotted line: LTE including $P_{\nu}=0.01 B_{\nu}(80 \mathrm{~K})+B_{\nu}(2.7 \mathrm{~K})$, dash-dotted line: analytic approximation of McKee et al. (1982); thick lines indicate a negative cooling rate that means a net heating of the gas.

dust emission is negligible, 3 . only the ground vibrational state contains a significant population. Assumptions 1 and 3 hold also for our calculations, but contrary to them, we included pumping by the infrared radiation field. Our calculations show that pumping due to thermal dust emission of the disk and the cosmic microwave background can be important for the CO population numbers (Kamp \& van Zadelhoff 2000). Comparing our cooling rates with IR pumping by the cosmic microwave background (Fig. 5 dashed lines) to the numerical fits of McKee et al. (1982) (Fig. 5 dash-dotted lines), they agree reasonable well within a factor $2.5 / 3$ for $T_{\text {gas }}=100 \mathrm{~K} / 20 \mathrm{~K}$. This is within the error which the authors claim for their fits.

Besides this good agreement between our calculations and those of McKee et al. (1982), Fig. 5 shows the effect of the IR radiation field $P_{\nu}$ (solid line: with $P_{\nu}=0.01 B_{\nu}(80 \mathrm{~K})+B_{\nu}(2.7 \mathrm{~K})$, dashed line: with $P_{\nu}=$ $B_{\nu}(2.7 \mathrm{~K})$, long-dashed line: $\left.P_{\nu}=B_{\nu}(80 \mathrm{~K})+B_{\nu}(2.7 \mathrm{~K})\right)$. 

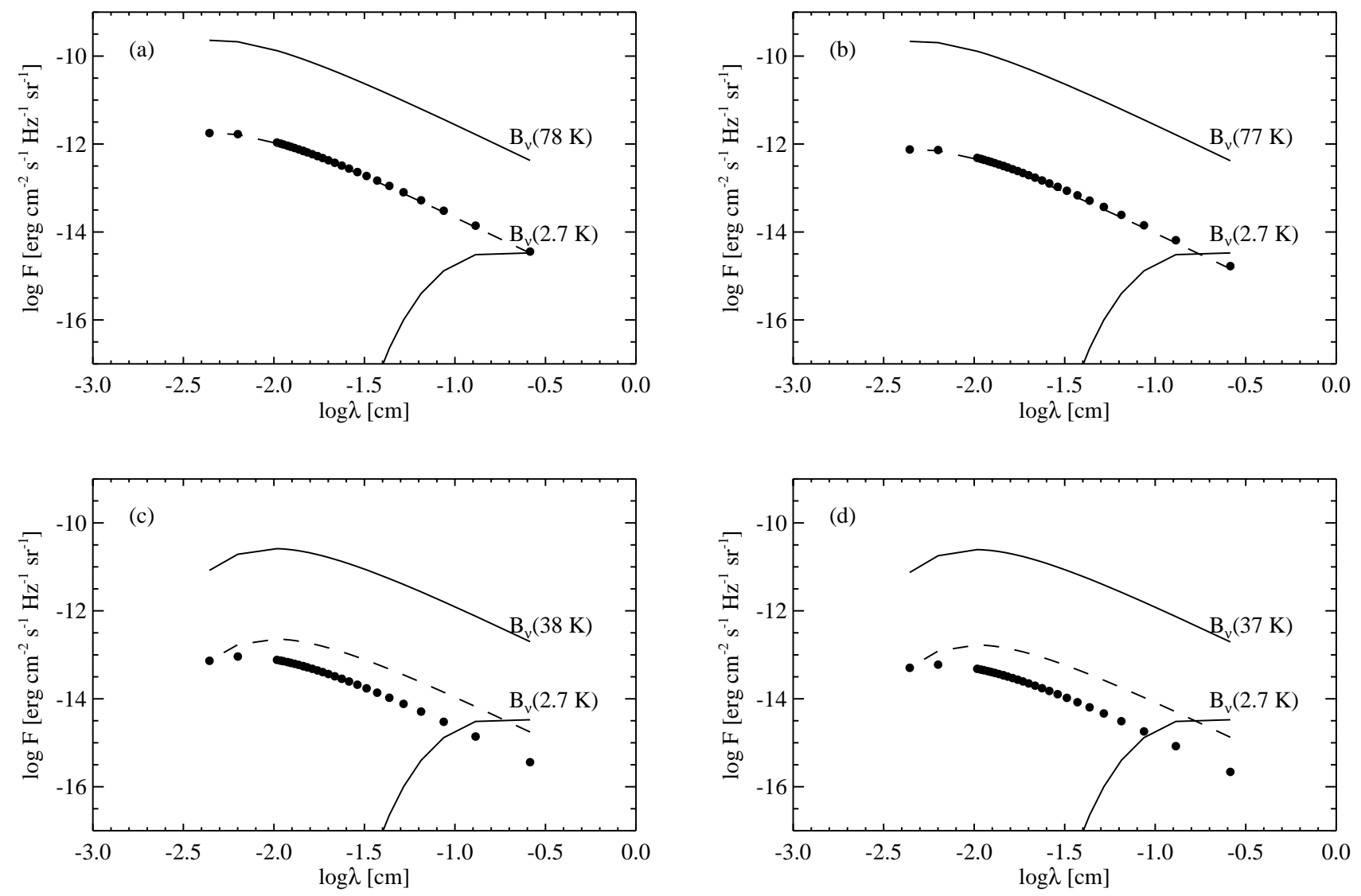

Fig. 6. Infrared radiation field for a $2 M_{\oplus} \beta$ Pictoris disk at four different grid points: a) $r=50$ AU, disk midplane, b) $r=50$ AU, $z=15 \mathrm{AU}, \mathbf{c}) r=307 \mathrm{AU}$, disk midplane, d) $r=307 \mathrm{AU}, z=92 \mathrm{AU}$. The black dots denote the radiation field due to the dust derived from Eq. (40). The upper solid curve shows the local approximation $P_{\nu}(r, z)=B_{\nu}\left(T_{\mathrm{d}}(r, z)\right)$, the lower one the cosmic microwave background $B_{\nu}(2.7 \mathrm{~K})$. The dashed curve shows the local approximation scaled to the shortest wavelength point.

At low particle densities the cooling rate turns into a heating rate for the two cases $P_{\nu}=0.01 B_{\nu}(80 \mathrm{~K})+B_{\nu}(2.7 \mathrm{~K})$ and $P_{\nu}=B_{\nu}(2.7 \mathrm{~K})$. Only at low temperatures and with a much stronger radiation field $P_{\nu}=B_{\nu}(80 \mathrm{~K})+B_{\nu}(2.7 \mathrm{~K})$ we find a net heating of the gas over the whole range of densities considered here. For the optically thin disk models presented here, Figs. 5 and 6 reveal that the pumping of the $\mathrm{CO}$ molecule by thermal dust emission is negligible for densities larger than $10^{3} \mathrm{~cm}^{-3}$ and that LTE can overestimate the cooling rates by a factor 3 to 10 even at high densities.

[C I] fine structure line cooling. Considering the chemical structure of the $\beta$ Pictoris disks (Kamp \& Bertoldi 2000) [C I] fine structure line cooling may become important due to the presence of neutral carbon. The calculation of the cooling rates makes use of the three level atom approximation from Hollenbach \& McKee (1989) $\mathrm{CI}\left({ }^{3} \mathrm{P}_{0},{ }^{3} \mathrm{P}_{1}\right.$, $\left.{ }^{3} \mathrm{P}_{2}\right)$. The respective cooling rates for the three lines at $609.2,229.9$, and $369.0 \mu \mathrm{m}$ are

$\Lambda_{9 / 10 / 11}=A_{i j} h \nu_{i j} n_{i} \quad \operatorname{erg} \mathrm{cm}^{-3} \mathrm{~s}^{-1}$.

The spontaneous transitions rates $A_{i j}$ are $7.9 \times 10^{-8}, 2.0 \times$ $10^{-14}$, and $2.7 \times 10^{-4}$. For the level population numbers we can assume LTE, because the gas densities in these disks are well above $2 \times 10^{3} \mathrm{~cm}^{-3}$, the critical density for LTE (see Table 8 of Hollenbach \& McKee 1989).

\section{The infrared radiation field}

We stressed already in Sect. 2.1 the importance of the infrared radiation field for the determination of the statistical equilibrium of oxygen and carbon monoxide. This radiation field consists of two components: one is the thermal emission of the large dust grains and the other is the $2.7 \mathrm{~K}$ cosmic microwave background.

Since the disks are optically thin, we can calculate the mean radiation field from the dust grains at any point $(r$, $z$ ) in the disk straight forwardly by integrating over the whole disk volume

$$
\begin{array}{r}
P_{\nu, \mathrm{d}}(r, z)=\frac{1}{8 \pi^{2}} \int_{0}^{\pi} \int_{-Z_{\mathrm{o}}}^{Z_{\mathrm{o}}} \int_{R_{\mathrm{i}}}^{R_{\mathrm{o}}} \frac{\pi a^{2}}{y^{2}} n_{\mathrm{d}}\left(r^{\prime}, z^{\prime}\right) \\
B_{\nu}\left(T_{\mathrm{d}}\left(r^{\prime}, z^{\prime}\right)\right) r^{\prime} \mathrm{d} \phi \mathrm{d} z^{\prime} \mathrm{d} r^{\prime},
\end{array}
$$

where $Z_{\mathrm{o}}, R_{\mathrm{i}}$ and $R_{\mathrm{o}}$ define the outer boundary of the disk model. The distance from $(r, z)$ to any other point $\left(r^{\prime}, z^{\prime}\right)$ in the disk is given by

$y=\sqrt{r^{2}+r^{\prime 2}-2 r r^{\prime} \cos \phi+\left(z^{\prime}-z\right)^{2}}$, 
Table 2. Molecular line data for the CO model molecule: upper level of the rotational lines $J \rightarrow J-1$, energy of the upper level, wavelengths of the lines, Einstein transition probabilities for spontaneous emission, and fits to the critical densities which hold for $10 \mathrm{~K}<T_{\text {gas }}<100 \mathrm{~K}$; the statistical weight of a level is $g=2 J+1$.

\begin{tabular}{rrrrl}
\hline$J$ & $E\left[\mathrm{~cm}^{-1}\right]$ & $\lambda[\mathrm{mm}]$ & $A_{i j}\left[\mathrm{~s}^{-1}\right]$ & $n_{\mathrm{cr}}\left[\mathrm{cm}^{-3}\right]$ \\
\hline 1 & 3.85 & 2.601 & $7.17 \times 10^{-8}$ & $5.0 \times 10^{3} T^{-0.66}$ \\
2 & 11.54 & 1.300 & $6.87 \times 10^{-7}$ & $1.9 \times 10^{4} T^{-0.45}$ \\
3 & 23.07 & 0.867 & $2.48 \times 10^{-6}$ & $4.6 \times 10^{4} T^{-0.35}$ \\
4 & 38.45 & 0.650 & $6.09 \times 10^{-6}$ & $8.3 \times 10^{4} T^{-0.28}$ \\
5 & 57.67 & 0.520 & $1.22 \times 10^{-5}$ & $1.3 \times 10^{5} T^{-0.22}$ \\
6 & 80.74 & 0.434 & $2.13 \times 10^{-5}$ & $2.0 \times 10^{5} T^{-0.18}$ \\
7 & 107.64 & 0.372 & $3.40 \times 10^{-5}$ & $2.9 \times 10^{5} T^{-0.16}$ \\
8 & 138.39 & 0.325 & $5.11 \times 10^{-5}$ & $4.1 \times 10^{5} T^{-0.14}$ \\
9 & 172.98 & 0.289 & $7.29 \times 10^{-5}$ & $5.5 \times 10^{5} T^{-0.12}$ \\
10 & 211.40 & 0.260 & $1.00 \times 10^{-4}$ & $7.2 \times 10^{5} T^{-0.11}$ \\
11 & 253.67 & 0.237 & $1.33 \times 10^{-4}$ & $9.2 \times 10^{5} T^{-0.10}$ \\
12 & 299.77 & 0.217 & $1.73 \times 10^{-4}$ & $1.1 \times 10^{6} T^{-0.09}$ \\
13 & 349.70 & 0.200 & $2.19 \times 10^{-4}$ & $1.4 \times 10^{6} T^{-0.08}$ \\
14 & 403.46 & 0.186 & $2.72 \times 10^{-4}$ & $1.7 \times 10^{6} T^{-0.075}$ \\
15 & 461.05 & 0.174 & $3.33 \times 10^{-4}$ & $2.0 \times 10^{6} T^{-0.07}$ \\
16 & 522.48 & 0.163 & $4.02 \times 10^{-4}$ & $2.4 \times 10^{6} T^{-0.065}$ \\
17 & 587.72 & 0.153 & $4.80 \times 10^{-4}$ & $2.8 \times 10^{6} T^{-0.06}$ \\
18 & 656.79 & 0.145 & $5.66 \times 10^{-4}$ & $3.2 \times 10^{6} T^{-0.055}$ \\
19 & 729.68 & 0.137 & $6.60 \times 10^{-4}$ & $3.7 \times 10^{6} T^{-0.05}$ \\
20 & 806.38 & 0.130 & $7.64 \times 10^{-4}$ & $4.2 \times 10^{6} T^{-0.045}$ \\
21 & 886.90 & 0.124 & $8.77 \times 10^{-4}$ & $4.7 \times 10^{6} T^{-0.04}$ \\
22 & 971.23 & 0.119 & $9.98 \times 10^{-4}$ & $5.3 \times 10^{6} T^{-0.035}$ \\
23 & 1059.37 & 0.113 & $1.13 \times 10^{-3}$ & $5.9 \times 10^{6} T^{-0.03}$ \\
24 & 1151.32 & 0.109 & $1.27 \times 10^{-3}$ & $6.5 \times 10^{6} T^{-0.025}$ \\
25 & 1247.06 & 0.104 & $1.42 \times 10^{-3}$ & $6.8 \times 10^{6}$ \\
\hline & & & &
\end{tabular}

and the dust particle density can be derived from the gas density

$n_{\mathrm{d}}=\delta_{\mathrm{dg}} n_{\mathrm{g}} \mu m_{\mathrm{H}} \frac{3}{4 \pi a^{3} \rho_{\text {grain }}} \mathrm{cm}^{-3}$,

where $\delta_{\mathrm{dg}}$ is the dust-to-gas mass ratio and $\rho_{\text {grain }}$ is the material density of the dust grains. The total infrared radiation field is then the sum of the two components

$P_{\nu}(r, z)=P_{\nu, \mathrm{d}}(r, z)+B_{\nu}(2.7 \mathrm{~K})$.

The dust particle density is too low to ensure a total $4 \pi$ coverage even in the innermost regions of the disk. For an oxygen atom sitting in the disk plane the surrounding medium is more or less a dark background with a lot of bright spots, the dust grains. Hence the local approximation $P_{\nu}(r, z)=B_{\nu}\left(T_{\mathrm{d}}(r, z)\right)$ completely fails. Figure 6 reveals the difference beween these two approaches for four different points in the disk. While in the inner regions of the disk the IR radiation field has at least the same temperature as the local gas, this is clearly not the case in the cool outer parts (Fig. 6). Moreover the radiation field cannot be represented by some kind of mean temperature scaled according to the solid angle of dust grains.

Black body grains with a size of $3 \mu \mathrm{m}$ have at $60 \mu \mathrm{m}$ an absorption efficiency of $Q_{\mathrm{abs}}=0.6$. The assumption of
Table 3. Standard model parameters.

\begin{tabular}{lll}
\hline Parameter & Name & Standard value \\
\hline grain size & $a$ & $3 \mu \mathrm{m}$ \\
grain density & $\rho_{\text {grain }}$ & $3 \mathrm{~g} \mathrm{~cm}^{-3}$ (silicates) \\
inner radius & $R_{\mathrm{i}}$ & $40 \mathrm{AU}$ \\
outer radius & $R_{\mathrm{O}}$ & $500 \mathrm{AU}$ \\
dust-to-gas mass ratio & $\delta_{\mathrm{dg}}$ & 0.01 \\
disk scale height & $H$ & 0.15 \\
fraction of $\mathrm{H}_{2}^{*}$ & $f_{\mathrm{H}_{2}^{*}}$ & $10^{-5}$ \\
UV dust ext. cross section & $\sigma_{\mathrm{UV}}$ & $2.34 \times 10^{-23} \mathrm{~cm}^{2}(\mathrm{H})^{-1}$ \\
carbon abundance & $\epsilon_{\mathrm{C}}$ & $1.4 \times 10^{-4}$ \\
oxygen abundance & $\epsilon_{\mathrm{O}}$ & $3.2 \times 10^{-4}$ \\
\hline
\end{tabular}

a black body radiation field is no longer valid when the dust temperature changes by more than $5 \%$ over an optical depth of 1 , which means a total column density of $7 \times 10^{22} \mathrm{~cm}^{-2}$. For the disk model described here, the assumption of a black body radiation field is valid at $50 \mathrm{AU}$ for total disk masses (between 40 and $500 \mathrm{AU}$ ) larger than $7.4 \times 10^{4} M_{\oplus}$ and at $100 \mathrm{AU}$ for masses larger than $4.2 \times 10^{5} M_{\oplus}$. These values are well above the disk masses considered here.

The infrared radiation field depends only on the density structure of the disk and on the dust temperature. Since the latter is fixed by the assumption of radiative equilibrium, it can be calculated in advance and does not change during the numerical calculation of the gas chemistry and gas temperature.

\section{The gas temperature: Numerical models}

The input parameters listed in Table 3 remain the same during the calculations presented here; we assume that the grains are composed of silicates. The only parameters changed are the disk mass and stellar radiation field. The models are calculated for two different disk masses, namely $2 M_{\oplus}$ and $0.2 M_{\oplus}$ and for the radiation fields of two prototype stars $\beta$ Pictoris and Vega, which represent the cool and the hot end in the range of A stars.

The radiation field of $\beta$ Pictoris is strong enough to blow out $3 \mu \mathrm{m}$ silicate grains, since these disks rotate with slightly subkeplerian velocity around the star. The motion of the dust particles will be much more complicated than described in Eq. (23) and the velocities will probably be somewhat smaller. But in order to bracket reality, we have carried out the calculations for two extreme cases: 1. "maximum drift velocity", 2. $v_{\text {drift }}=0$. We will first discuss the numerical results including $v_{\text {drift }}=v_{\text {drift }}^{\max }$ and afterwards the results without drift velocity heating.

\section{1. $\beta$ Pictoris: $v_{\text {drift }}=v_{\text {drift }}^{\max }$}

Drift velocity heating dominates throughout the whole disk independent of the disk mass in the $\beta$ Pictoris models. The most important cooling processes for the $2 M_{\oplus}$ model are illustrated in Fig. 7. Since the stellar radiation field is too weak to efficiently dissociate carbon monoxide 
(Kamp \& Bertoldi 2000), the CO rotational lines are the most efficient coolant in the inner parts of the disk. Using the laboratory data for freezing out of $\mathrm{CO}$ on water ice surfaces (Sandford \& Allamandola 1990), at $T_{\text {dust }}<50 \mathrm{~K}$ all $\mathrm{CO}$ is incorporated in ice mantles around cold dust grains (Kamp \& Bertoldi 2000); hence [O I] fine structure cooling dominates in those parts. In the uppermost layers of the disk from inside to outside [O I], [C II] and then $\mathrm{H}_{2}$ line cooling are the most important cooling processes. By lowering the disk mass by a factor ten (see Fig. 8) the dominant processes become very similar to those found in the upper layers of the more massive disk model. The global picture does not change much, except that now [C II] and $\mathrm{H}_{2}$ line cooling become more important compared to [O I] cooling.
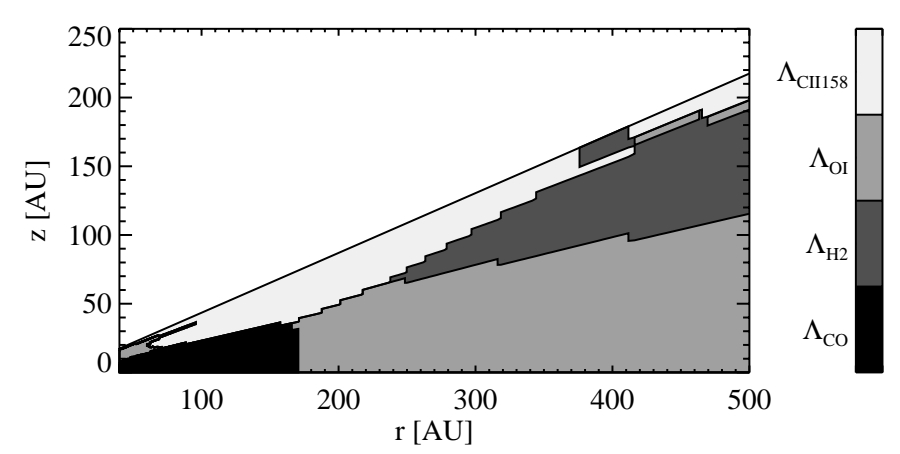

Fig. 7. The most important cooling processes in the $\beta$ Pictoris $2 M_{\oplus}$ disk model including all described heating and cooling processes (the bar displays only the relevant processes).

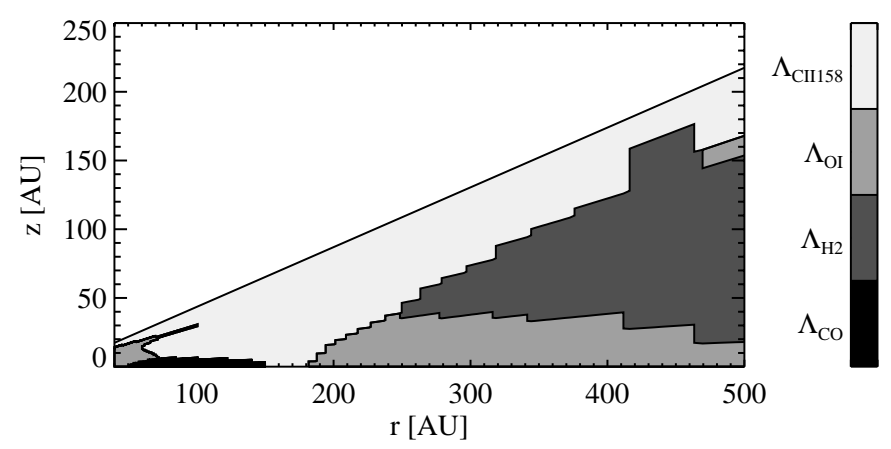

Fig. 8. The most important cooling processes in the $\beta$ Pictoris $0.2 M_{\oplus}$ disk model including all described heating and cooling processes (the bar displays only the relevant processes).

The resulting temperature structure of both disk models is shown in Figs. 9 and 10. Due to the strong drift velocity heating, the gas temperature of the disk is always larger than the dust temperature, except for a small core, which is below $10 \mathrm{~K}$ at $100 \mathrm{AU}$ in the $2 M_{\oplus}$ model. In both models we find a small hot surface layer in the inner parts of the disk. We will show later in Sect. 6 that the total mass contained in this hot layer is less than $1 \%$ of the total disk mass.

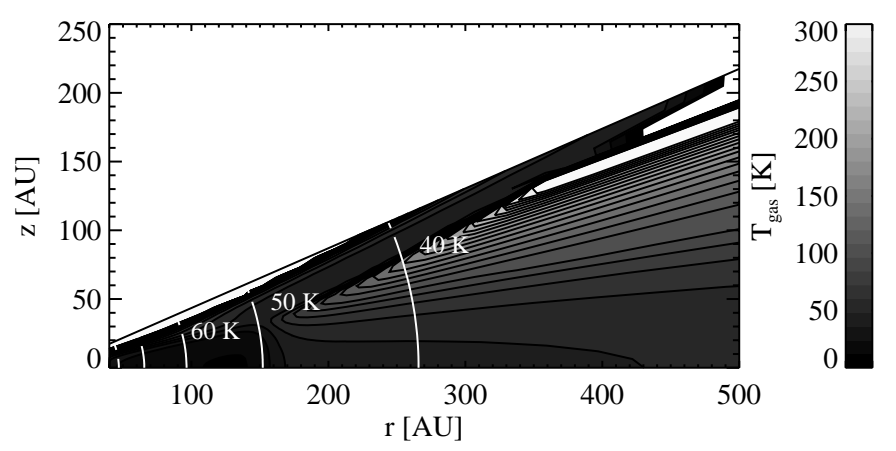

Fig. 9. Temperature structure of the $\beta$ Pictoris $2 M_{\oplus}$ disk model including all described heating and cooling processes: the grey colors show the gas temperature as noted on the scale at the right hand side, while the overlayed white contour lines show the dust temperature in steps of $10 \mathrm{~K}$.
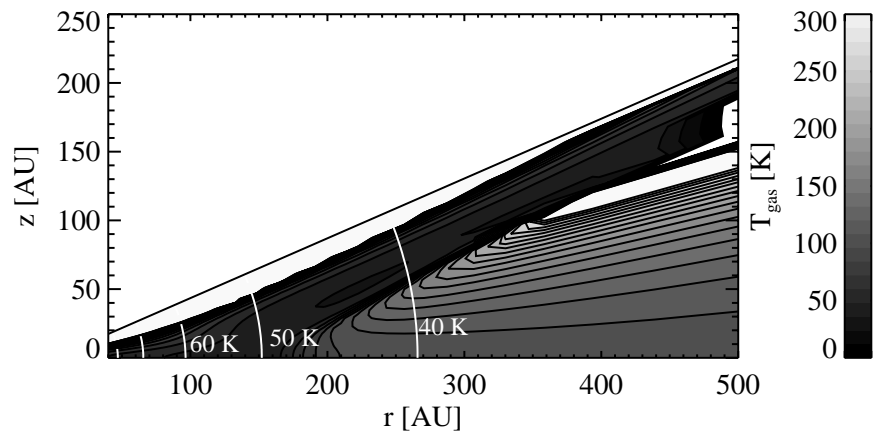

Fig. 10. Same as 9 , but for a $\beta$ Pictoris $0.2 M_{\oplus}$ disk.

\section{2. $\beta$ Pictoris: $v_{\text {drift }}=0$}

If we neglect the drift velocity heating in the $\beta$ Pictoris disk, cosmic ray heating becomes important in large parts of the disk (see Fig. 11). In the inner regions of the disk plane, the gas is heated by pumping of the OI levels. Looking at the upper layers, we find that in the inner regions heating by $\mathrm{H}_{2}$ formation dominates, while outwards $\mathrm{H}_{2}$ dissociation is more important. On the other hand Fig. 12 shows, that [C II] fine-structure cooling dominates in the upper disk layers, where carbon is not bound in CO. While in the innermost parts CO rotational line cooling contributes largely to the total cooling rate, more outwards, where $\mathrm{CO}$ is frozen onto the cool dust grains, [O I] fine structure cooling determines the gas temperature.

The heating/cooling processes for the $0.2 M_{\oplus}$ model are shown in Figs. 13 and 14. Cosmic ray heating is now also important in the uppermost low density disk layers. Between the two regions of cosmic ray heating, the gas is heated by $\mathrm{H}_{2}$ dissociation, while in the inner region of the disk $\mathrm{H}_{2}$ formation is more important. The cooling rates show a similar behaviour as for the $2 M_{\oplus}$ model except that $\mathrm{CO}$ rotational line cooling and [O I] fine structure cooling are now more concentrated towards the disk midplane.

Without any drift velocity heating, the gas in the disks becomes extremely cool, $T_{\text {gas }}<60 \mathrm{~K}$ (Figs. 15 and 16). Note that one of the main heating sources, namely heating 

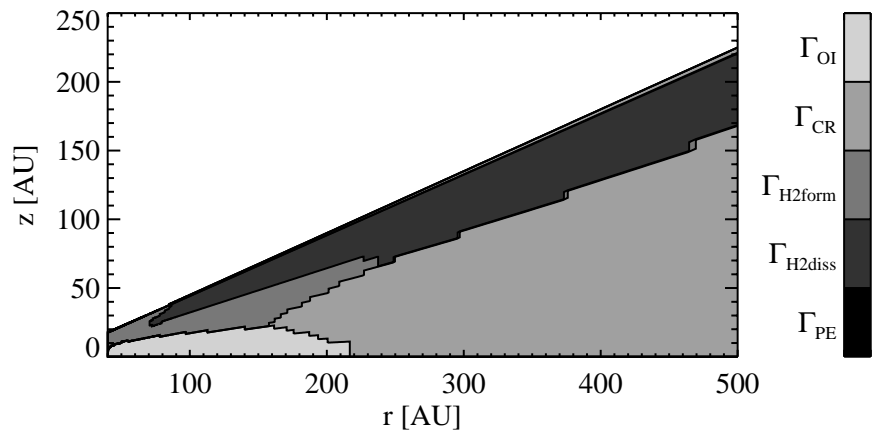

Fig. 11. The most important heating processes in the $\beta$ Pictoris $2 M_{\oplus}$ disk model including all described heating and cooling processes except the heating due to the drift velocity of grains through the gas (the bar displays only the relevant processes).
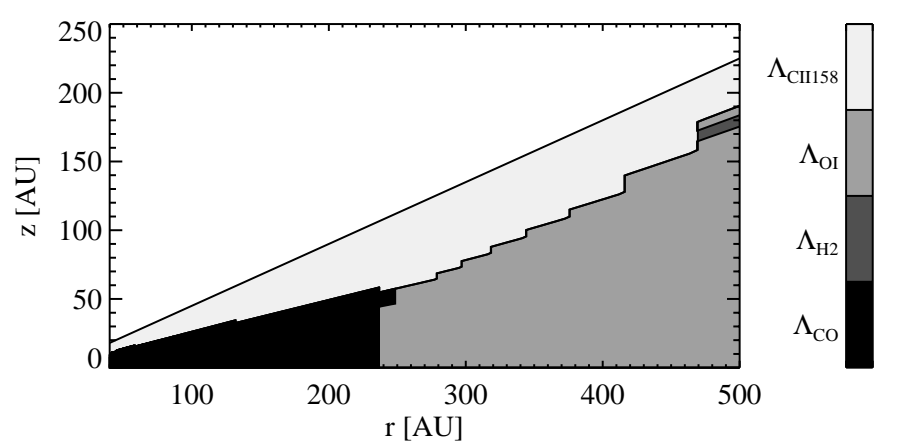

Fig. 12. The most important cooling processes in the $\beta$ Pictoris $2 M_{\oplus}$ disk model including all described heating and cooling processes except the heating due to the drift velocity of grains through the gas (the bar displays only the relevant processes).

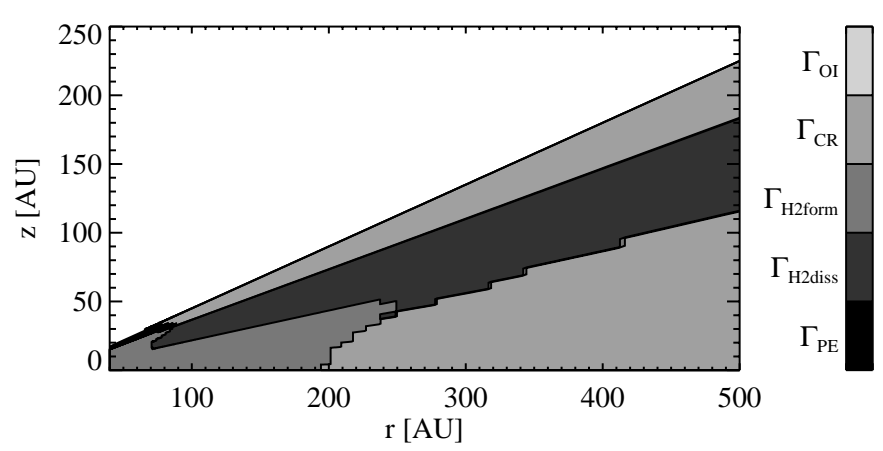

Fig. 13. The most important heating processes in the $\beta$ Pictoris $0.2 M_{\oplus}$ disk model including all described heating and cooling processes except the heating due to the drift velocity of grains through the gas (the bar displays only the relevant processes).

by $\mathrm{H}_{2}$ formation, is not as efficient as in molecular clouds, because the dust grains used in our disks are much larger than in the ISM, and hence the dust formation rate is lower (Kamp \& Bertoldi 2000).

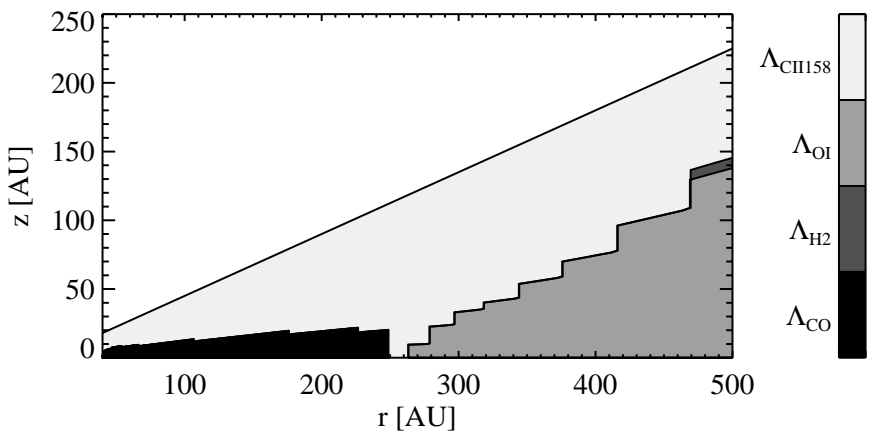

Fig. 14. The most important cooling processes in the $\beta$ Pictoris $0.2 M_{\oplus}$ disk model including all described heating and cooling processes except the heating due to the drift velocity of grains through the gas (the bar displays only the relevant processes).

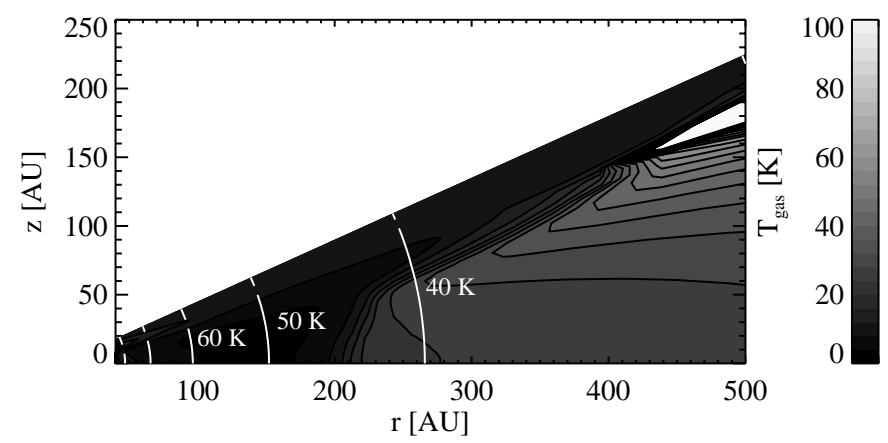

Fig. 15. Temperature structure of the $\beta$ Pictoris $2 M_{\oplus}$ disk model including all described heating and cooling processes except the heating due to the drift velocity of grains through the gas: the grey colors show the gas temperature as noted on the scale at the right hand side, while the overlayed white contour lines show the dust temperature in steps of $10 \mathrm{~K}$.
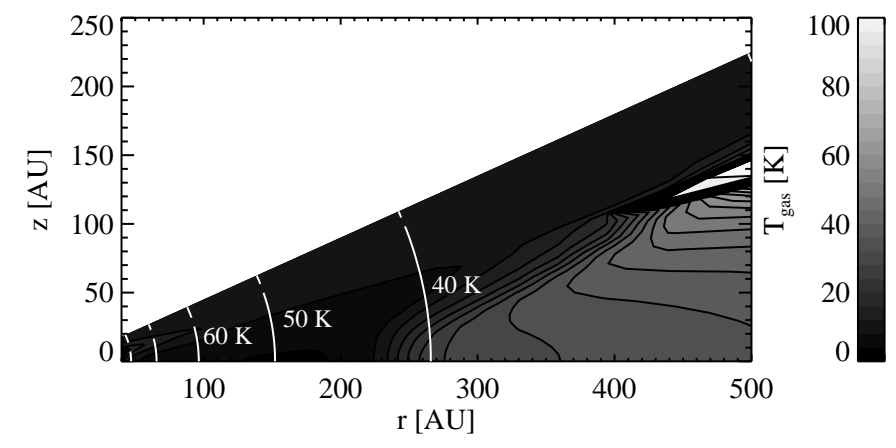

Fig. 16. Same as 15 , but for a $\beta$ Pictoris $0.2 M_{\oplus}$ disk.

\subsection{Vega: $v_{\text {drift }}=v_{\text {drift }}^{\max }$}

Contrary to the $\beta$ Pictoris model, CO is mainly photodissociated in the Vega disks and hence CO rotational line cooling is not important. Instead the main coolants are $\mathrm{O}$ I and $\mathrm{C}$ II.

Similar to the $\beta$ Pictoris models, drift velocity heating is the main energy input for the gas. Figure 17 shows, that [O I] (dense inner parts) and [C II] $157.7 \mu \mathrm{m}$ (outer parts) fine structure lines are the most important cooling processes of the $2 M_{\oplus}$ Vega model. 

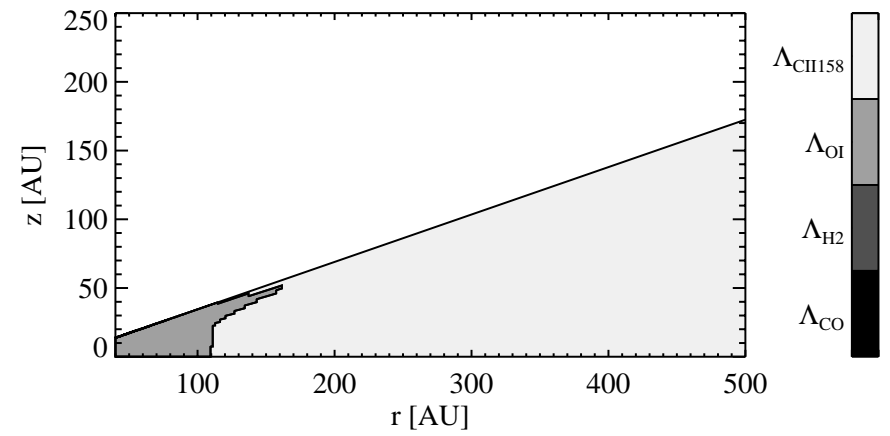

Fig. 17. The most important cooling processes in the Vega $2 M_{\oplus}$ disk model including all described heating and cooling processes (the bar displays only the relevant processes).
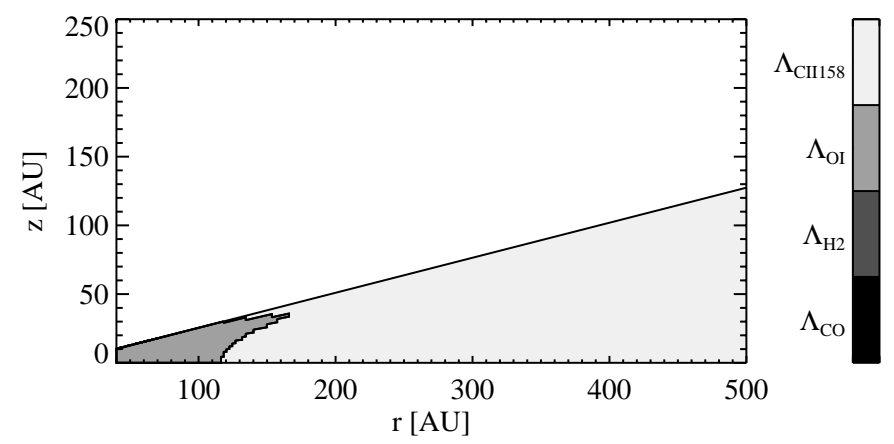

Fig. 18. The most important cooling processes in the Vega $0.2 M_{\oplus}$ disk model including all described heating and cooling processes (the bar displays only the relevant processes).

If we reduce the density in the disks by a factor 10 (Fig. 18), the most important cooling processes remain the same.

Figure 19 reveals that the gas temperatures throughout most of the $2 M_{\oplus}$ disk model stay well below $100 \mathrm{~K}$. Nevertheless the heating due to the drift velocity of dust grains through the gas leads to a hot surface layer, especially in the inner parts of the disk. Due to the lower particle densities in the $0.2 M_{\oplus}$ disk model this hot surface layer reaches deeper into the disk, leading to temperatures above $100 \mathrm{~K}$ even in the disk midplane. Most of the outer regions of the disk have nevertheless gas temperatures below $100 \mathrm{~K}$ (Fig. 20 note the different temperature scale).

\subsection{Vega: $v_{\text {drift }}=0$}

If the drift velocity is turned off, $\mathrm{H}_{2}$ dissociation and photoelectric heating take over (Figs. 21 and 23). Throughout most of the $2 M_{\oplus}$ disk model $\mathrm{H}_{2}$ photodissociation is the main heating source. Only in the uppermost layers photoelectric heating by the micron-sized grains become important. As already pointed out in Sect. 2.1, due to the large size of the dust grains, the photoelectric heating rate is 2 to 3 orders of magnitude lower than in molecular clouds with typical ISM grains. The main energy loss for this disk model occurs via the [C II] $157.7 \mu \mathrm{m}$ fine structure line.

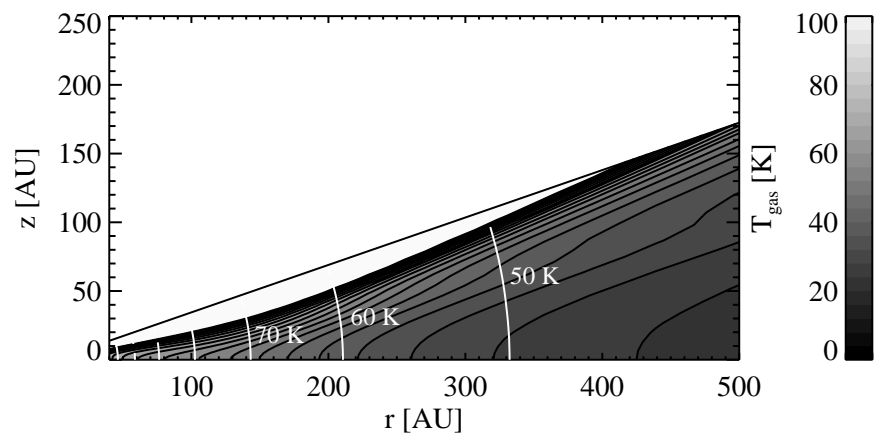

Fig. 19. Temperature structure of the Vega $2 M_{\oplus}$ disk model including all described heating and cooling processes: the grey colors show the gas temperature as noted on the scale at the right hand side, while the overlayed white contour lines show the dust temperature in steps of $10 \mathrm{~K}$.

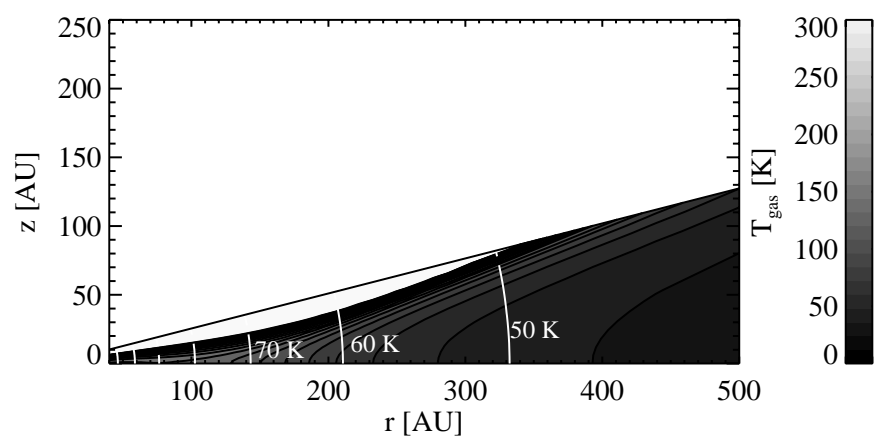

Fig. 20. Same as Fig. 19, but for a $0.2 M_{\oplus}$ disk.

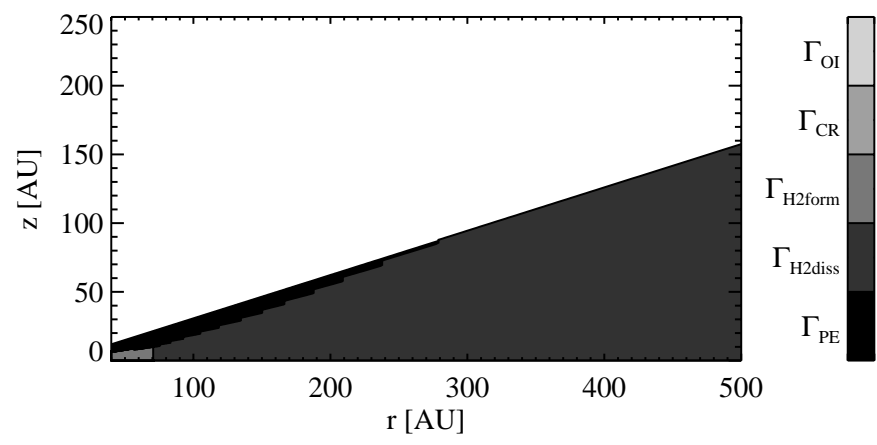

Fig. 21. The most important heating processes in the Vega $2 M_{\oplus}$ disk model including all described heating and cooling processes except the heating due to the drift velocity of grains through the gas (the bar displays only the relevant processes).

Only in the densest parts of the disk midplane the [O I] fine structure lines are more efficient in radiating away the energy (Fig. 22).

If we now reduce the disk mass by a factor 10 , the [C II] $157.7 \mu \mathrm{m}$ fine structure line is the sole remaining cooling process (see Fig. 24). On the other hand, Fig. 23 shows that the heating takes place via ejection of photoelectrons from the large dust grains (inner part of the modelled disk) and $\mathrm{H}_{2}$ photodissociation (outer parts of the disk). 

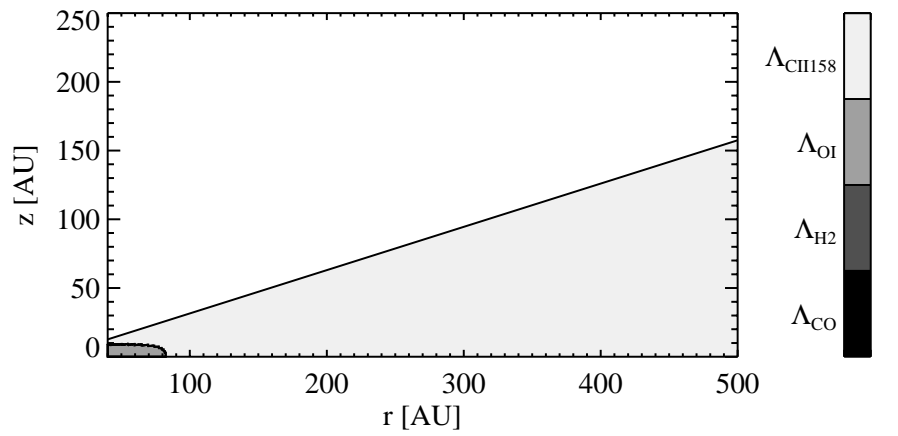

Fig. 22. The most important cooling processes in the Vega $2 M_{\oplus}$ disk model including all described heating and cooling processes except the heating due to the drift velocity of grains through the gas (the bar displays only the relevant processes).

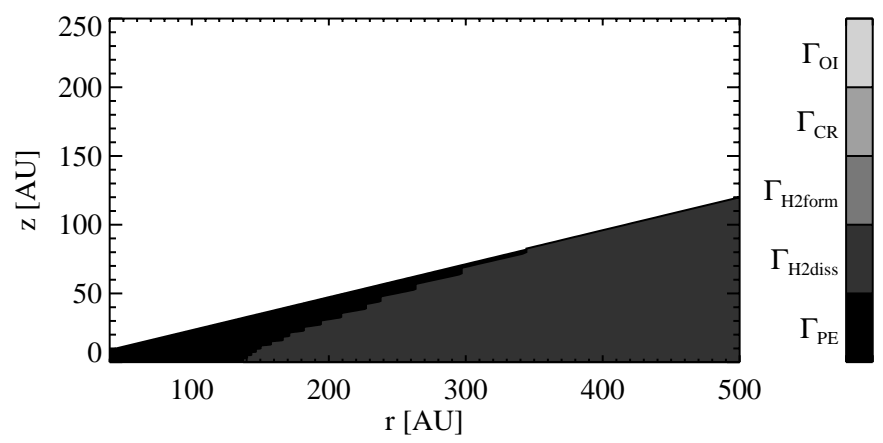

Fig. 23. The most important heating processes in the Vega $0.2 M_{\oplus}$ disk model including all described heating and cooling processes except the heating due to the drift velocity of grains through the gas (the bar displays only the relevant processes).

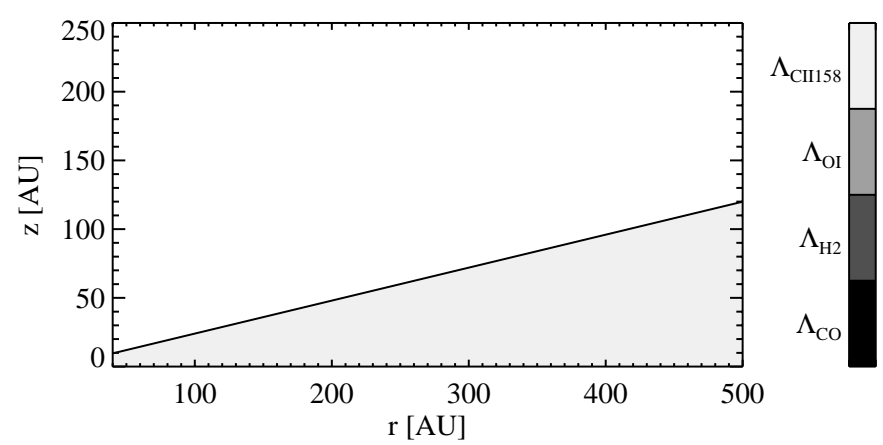

Fig. 24. The most important cooling processes in the Vega $0.2 M_{\oplus}$ disk model including all described heating and cooling processes except the heating due to the drift velocity of grains through the gas (the bar displays only the relevant processes).

The resulting temperature structure of both disk models, the $2 M_{\oplus}$ and the $0.2 M_{\oplus}$ disk model, is shown in Figs. 25 and 26. The hot surface layers, which we found for the models including the grain drift velocity, have now vanished. The whole disk models have moderate temperatures between 10 and $100 \mathrm{~K}$. Even though the gas temperatures are in the same range as the dust temperatures, they do not show the same structuring in the disk models. While the dust temperature depends only on the distance from the star, the gas temperature depends on the particle densities and chemical structure of the disk.

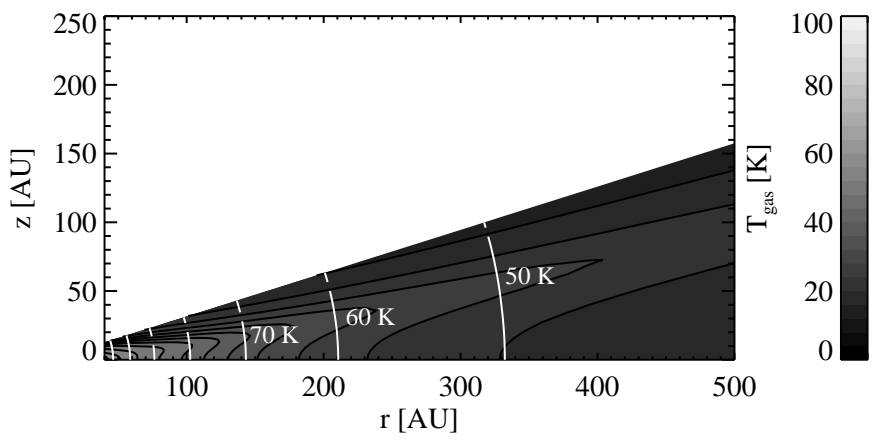

Fig. 25. Temperature structure of the Vega $2 M_{\oplus}$ disk model including all described heating and cooling processes except the heating due to the drift velocity of grains through the gas: the grey colors show the gas temperature as noted on the scale at the right hand side, while the overlayed white contour lines show the dust temperature in steps of $10 \mathrm{~K}$.

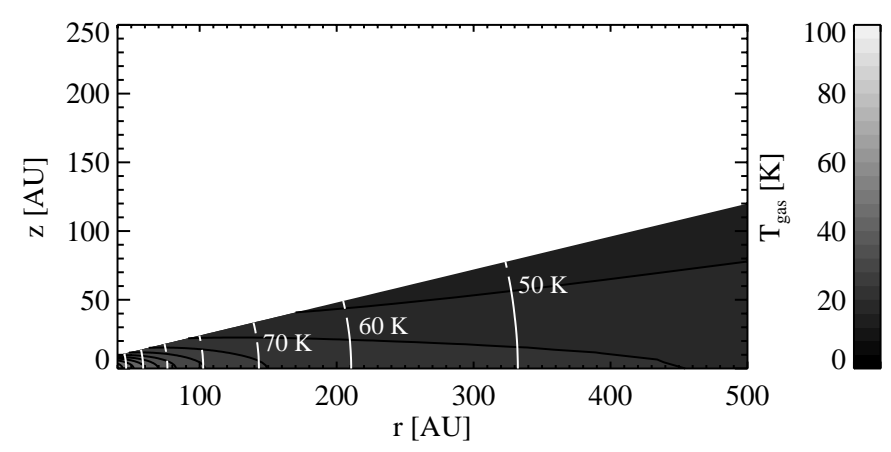

Fig. 26. Same as Fig. 25, but for the Vega $0.2 M_{\oplus}$ disk model.

\section{The gas temperature: Analytical approximations}

In the inner core of the $2 M_{\oplus} \beta$ Pictoris disk all carbon is in the form of $\mathrm{CO}$, hence radiation loss by the rotational lines of CO is the main cooling process. On the other hand the main heating process is gas heating due to the drift of dust grains through the gas.

In Sect. 2.1 we find that the analytical formula of McKee et al. (1982) is a good approximation for the detailed statistical equilibrium $\mathrm{CO}$ cooling rate, even at low temperatures. The density is always larger than the critical density $n_{\text {cr }}$ of McKee et al. (1982)

$n_{\mathrm{cr}}=1.86 \times 10^{4} T^{0.75} \mathrm{~cm}^{-3}$

which is a fit parameter in their formula and therefore different from the critical densities derived in this paper for each CO rotational line. We equate their Eq. (5.5a) modified by a factor of 0.5 (see Fig. 5) to our Eq. (27). This gives

$\tilde{T}_{\text {gas }}=\sqrt{256.6\left(\frac{3.7 \times 10^{-4}}{\epsilon_{\mathrm{CO}}}\right) \delta_{\mathrm{dg}} \rho_{\mathrm{tot}} \frac{v_{\mathrm{drift}}^{3}}{a \rho_{\text {grain }}}} \mathrm{K}$,

where $\epsilon_{\mathrm{CO}}=n_{\mathrm{CO}} / n_{\text {tot }}$ is the $\mathrm{CO}$ abundance, $\delta_{\mathrm{dg}}$ the dustto-gas mass ratio, $\rho_{\text {tot }}$ the total gas density, and $a$ and $\rho_{\text {grain }}$ are the grain radius and the material density of the dust grains respectively. Figure 27 shows the relative error 

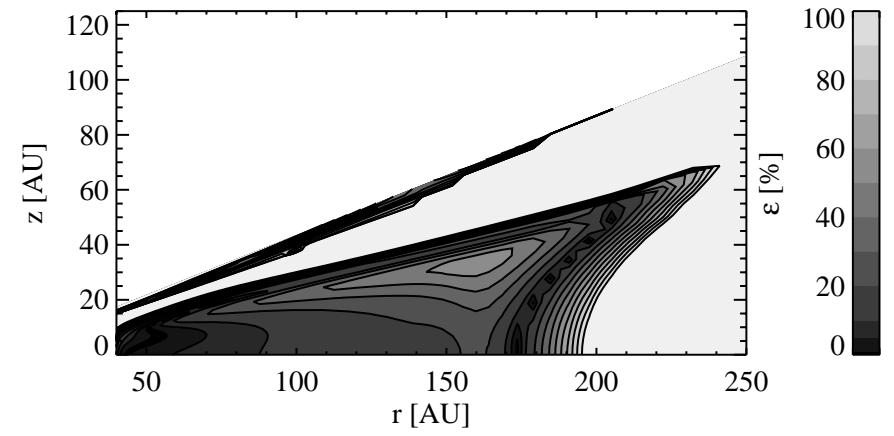

Fig. 27. Relative error of the analytical approximation for the gas temperature given in Eq. (45) in the inner parts of the $\beta$ Pictoris $2 M_{\oplus}$ model: $\epsilon=\left(\tilde{T}_{\text {gas }}-T_{\text {gas }}\right) / T_{\text {gas }}$ in $\%$.

$\left(\tilde{T}_{\text {gas }}-T_{\text {gas }}\right) / T_{\text {gas }}$ of this approximation throughout the inner disk regions.

$\mathrm{CO}$ is photodissociated in the Vega disks and furthermore carbon is mainly ionized. In the presence of drift velocity heating, we can thus make the simplifying approximation $\Lambda_{2}=\Gamma_{7},[\mathrm{CII}]$ fine structure line cooling equals drift velocity heating for the upper disk layers. For low gas temperatures the population number of the upper C II level is approximately $2 \times \mathrm{e}^{-91.98 / T}$. This leads to the expression

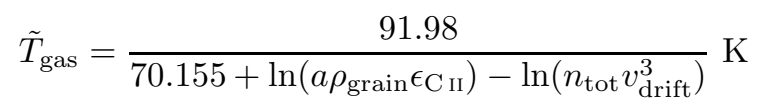

for the approximated gas temperature. Figure 28 illustrates that this approximation can be used throughout huge parts of the Vega 2.0 $M_{\oplus}$ disk model. Moreover it holds in the cool outer regions of the Vega $0.2 M_{\oplus}$ disk model.

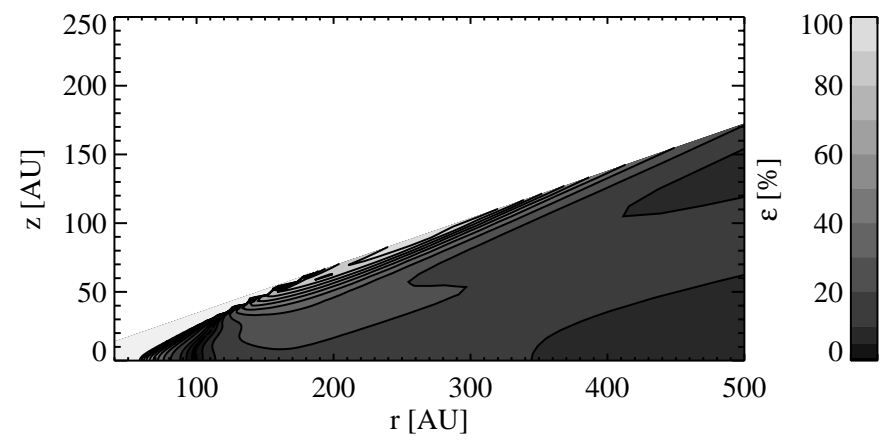

Fig. 28. Relative error of the analytical approximation for the gas temperature given in Eq. (46) in the Vega 2.0 $M_{\oplus}$ model: $\epsilon=\left(\tilde{T}_{\text {gas }}-T_{\text {gas }}\right) / T_{\text {gas }}$ in $\%$.

If we neglect drift velocity heating in these models, we can approximate the gas temperature in the inner parts of the $0.2 M_{\oplus}$ model by simply assuming $\Lambda_{2}=\Gamma_{1}$, [C II] fine structure line cooling equals photoelectric heating. For low gas temperatures we derive

$\tilde{T}_{\text {gas }}=\frac{91.98}{18.965+\ln \epsilon_{\text {CII }}-\ln \chi} \mathrm{K}$.

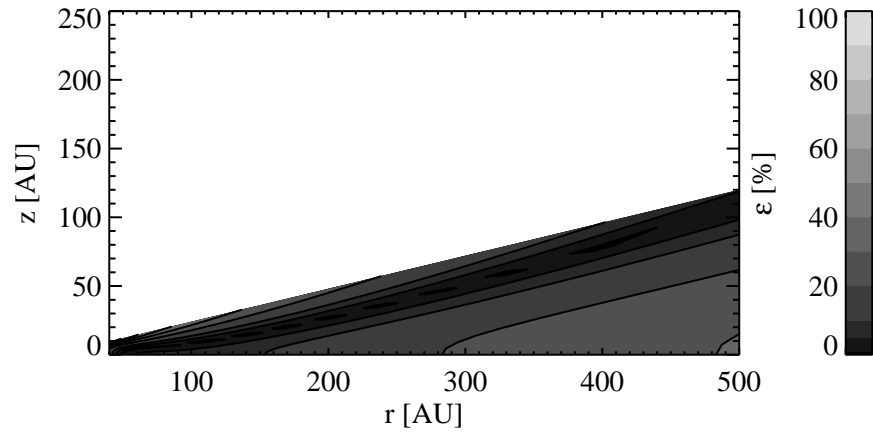

Fig. 29. Relative error of the analytical approximation for the gas temperature given in Eq. (47) in the Vega $0.2 M_{\oplus}$ model: $\epsilon=\left(\tilde{T}_{\text {gas }}-T_{\text {gas }}\right) / T_{\text {gas }}$ in $\%$.

This approximation allows to calculate the disk temperature throughout the whole Vega $0.2 M_{\oplus}$ model with an accuracy better than about $25 \%$ (Fig. 29).

\section{The gas chemistry}

In order to investigate the temperature dependence of our chemical network, we calculate the same models as discussed in Sect. 4 but with the assumption $T_{\text {gas }}=T_{\text {dust }}$.

To illustrate the effect of the gas temperature on the disk chemistry, we show in Figs. 30 and 31 the CO abundances in the $\beta$ Pictoris $2 M_{\oplus}$ model with the approximation $T_{\text {gas }}=T_{\text {dust }}$ and with $T_{\text {gas }}$ derived from the heating/cooling balance. The difference in $\mathrm{CO}$ abundance is very small and the $\mathrm{CO}$ mass in these models is $1.61 \times 10^{-3}$ and $1.71 \times 10^{-3} M_{\oplus}$ respectively.

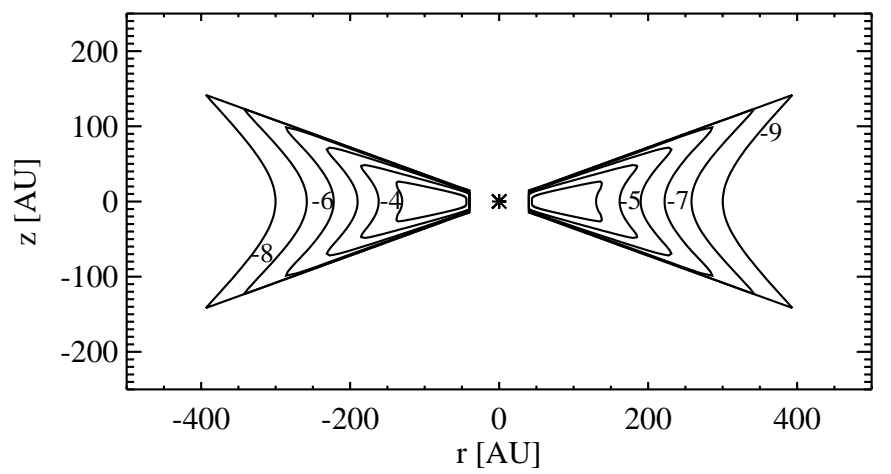

Fig. 30. $\log n_{\mathrm{CO}} / n_{\text {tot }}$ in the $2 M_{\oplus}$ model of $\beta$ Pictoris assuming $T_{\text {gas }}=T_{\text {dust }}$.

This shows that gas chemistry does not change very much for gas temperatures below $300 \mathrm{~K}$. Above this threshold reactions with an activation barrier start to become important and they can change the overall chemistry. Table 4 gives the mass of material in certain temperature ranges. The mass of material hotter than $300 \mathrm{~K}$ is negligible in our disk models except in the $0.2 M_{\oplus}$ Vega model with drift velocity heating, where it is $5 \%$ of the total mass. On the other hand a large amount of the total mass can have gas temperatures below $30 \mathrm{~K}$. Note that a 


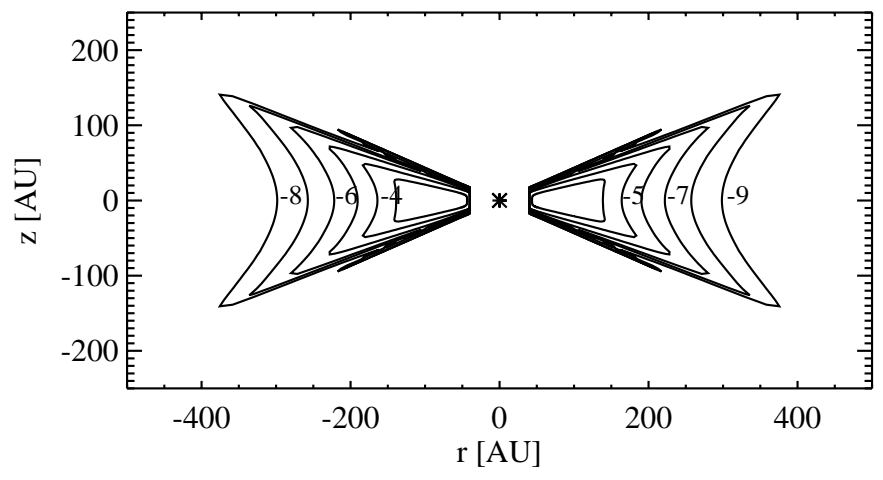

Fig. 31. $\log n_{\mathrm{CO}} / n_{\text {tot }}$ in the $2 M_{\oplus}$ model of $\beta$ Pictoris deriving $T_{\text {gas }}$ from the heating/cooling balance including all relevant processes $\left(v_{\mathrm{drift}}=v_{\mathrm{drift}}^{\max }\right)$.

low gas temperature is a necessary, but not sufficient criterion for freezing out of molecules. For this process the dust temperature plays a crucial role. The mass of material hotter than $100 \mathrm{~K}$ is the mass that can be traced by $\mathrm{H}_{2}$ lines assuming the disks to consist of purely molecular hydrogen. This is at least for the $\beta$ Pictoris models with the heating due to the drift velocity of dust grains a significant percentage of the total disk mass.

Table 4. Overview of disk masses in a certain temperature range for all models $\left(\Gamma_{7}\right.$ denotes heating due to the drift velocity of dust grains through the gas); all masses are given in $M_{\oplus}$.

\begin{tabular}{llllll}
\hline $\begin{array}{l}\text { central } \\
\text { star }\end{array}$ & $\Gamma_{7}$ & $\begin{array}{l}M \\
\text { total }\end{array}$ & $\begin{array}{l}M \\
T_{\mathrm{g}}>300 \mathrm{~K}\end{array}$ & $\begin{array}{l}M \\
T_{\mathrm{g}}>100 \mathrm{~K}\end{array}$ & $\begin{array}{l}M \\
T_{\mathrm{g}}<30 \mathrm{~K}\end{array}$ \\
\hline$\beta$ Pic & on & 2.0 & $7 \times 10^{-4}$ & 0.04 & 0.71 \\
$\beta$ Pic & on & 0.2 & $10^{-3}$ & 0.10 & $6 \times 10^{-5}$ \\
$\beta$ Pic & off & 2.0 & 0.0 & $5 \times 10^{-5}$ & 1.96 \\
$\beta$ Pic & off & 0.2 & 0.0 & $7 \times 10^{-5}$ & 0.14 \\
Vega & on & 2.0 & 0.01 & 0.05 & 0.52 \\
Vega & on & 0.2 & 0.01 & 0.09 & 0.00 \\
Vega & off & 2.0 & 0.0 & 0.0 & 1.17 \\
Vega & off & 0.2 & 0.0 & 0.0 & 0.16 \\
\hline
\end{tabular}

\section{Discussion}

Throughout the whole paper we discussed the two extreme cases for the dust grain drift velocity. But is there not a better and more accurate way to derive the drift velocity?

Klahr \& Lin (2001) solved the equation of motion for dust grains rotating in a gaseous disk around a central star. For the implementation of the hydrodynamical drag, it is assumed that the dust grains are in the Epstein regime, which means that their friction time scale is much smaller than their orbital timescale. Unfortunately this is not fullfilled for the $3 \mu \mathrm{m}$ grains in our disk models. Nevertheless using their Eq. (17) for the radial drift velocity, we end up with drift velocities which are a factor $2-5$ lower than the ones we applied in our models. Since the drift velocity enters cubic in the respective heating term, this would lead to heating rates that are up to a factor 125 smaller than the ones assumed in our $v_{\text {drift }}=v_{\text {drift }}^{\max }$ models. But if we use $2 \mu \mathrm{m}$ grains instead of $3 \mu \mathrm{m}$ grains, we end up with drift velocities that are even somewhat larger than ours.

To summarize, it is obvious that the heating term due to the drift velocity of dust grains through the gas depends strongly on the exact drift velocity. Unfortunately the radial drift velocity depends also strongly on particle size, which we cannot fix exactly. Moreover the implementation of a more realistic hydrodynamical drag term may change the resulting radial drift velocity considerably. In any case our two approaches $v_{\mathrm{drift}}=v_{\mathrm{drift}}^{\max }$ and $v_{\mathrm{drift}}=0$ will bracket the real solution.

\section{Conclusion}

The models presented in this paper are a link between the PDR models for dark molecular clouds (Tielens \& Hollenbach 1985; Sternberg \& Dalgarno 1989) and massive flaring disk models used for $\mathrm{T}$ Tauri stars and Herbig Ae/Be stars (Aikawa et al. 1996; Chiang \& Goldreich 1997; D'Alessio et al. 1998). The optically thin non-flaring disk models for young A stars presented by Kamp \& Bertoldi (2000) are extended to self-consistently include the calculation of the gas temperature from a detailed heating/cooling balance.

The model calculations reveal that gas and dust temperatures in these disks are completely different. Nevertheless there is still a coupling between them due to the IR pumping of $\mathrm{O}$ I fine structure and $\mathrm{CO}$ rotational levels. Since the disks are optically thin, this coupling does not result in $T_{\text {gas }}=T_{\text {dust }}$, but instead in level population numbers that adjust to the local IR radiation field, which is several orders of magnitude below the local Planck function.

Since atomic and molecular transition probabilities are known with sufficient accuracy, the main uncertainty in the calculation of the gas temperature are the physical parameters of the dust grains, like the size and the composition. Except for the bright star $\beta$ Pictoris, where the silicate feature at $10 \mu \mathrm{m}$ is clearly detected (Telesco \& Knacke 1991; Aitken et al. 1993), we lack observations that could constrain the composition of the dust grains in these disks.

The chemical structure of these disks does not change significantly, when we drop the approximation $T_{\text {gas }}=$ $T_{\text {dust }}$ and calculate the gas temperature from a detailed heating/cooling balance. Hence the main results of Kamp \& Bertoldi (2000) concerning the CO abundances in these disks do not change.

The gas temperature, the infrared radiation field and the statistical equilibrium calculations are crucial for the calculation of emission lines from these models. The results of the SE calculations show that level population numbers can easily be orders of magnitude different from thermodynamical equilibrium. 
We will present emission line profiles and possible tracers of the gas in these disks in a forthcoming paper.

Acknowledgements. We are indebted to F. Bertoldi and H. Holweger for many helpful discussions. Furthermore we benefited greately from discussions with E. van Dishoeck on the heating/cooling network. We thank E. van Dishoeck, Xander Tielens and David Hollenbach for a critical reading of the manuscript and helpful comments. This research has been supported by the "Deutsche Forschungsgesellschaft" under grant Ho 596/35-2 and by a Marie Curie Fellowship of the European Community programme "Improving Human Potential" under contract number MCFI-1999-00734.

\section{References}

Aikawa, Y., Miyama, S. M., Nakano, T., \& Umebayashi, T. 1996, ApJ, 467, 684

Aitken, D. K., Moore, T. J. T., Roche, P. F., Smith, C. H., \& Wright, C. M. 1993, MNRAS, 265, L41

Artymowicz, P. 1988, ApJ, 335, L79

Artymowicz, P., \& Clampin, M. 1997, ApJ, 490, 863

Augereau, J. C., Lagrange, A. M., Mouillet, D., \& Ménard, F. 2001, A\&A, 365, 78

Backman, D., \& Paresce, F. 1993, in Protostars \& Planets III, ed. E. H. Levy, \& J. I. Lunine (University of Arizona Press), 1253

Bakes, E. L. O., \& Tielens, A. G. G. M. 1994, ApJ, 427, 822

Bell, K. L., Berrington, K. A., \& Thomas, M. R. J. 1998, MNRAS, 293, L83

Black, J. H. 1987, in Interstellar Processes, ed. D. J. Hollenbach, \& H. A. Thronsen (D. Reidel Publishing Company), 731

Black, J. H., \& Dalgarno, A. 1976, ApJ, 203, 132

Burke, J. R., \& Hollenbach, D. J. 1983, ApJ, 265, 223

Chiang, E. I., \& Goldreich, P. 1997, ApJ, 490, 368

Chini, R., Krügel, E., \& Kreysa, E. 1990, A\&A, 227, L5

Clavel, J., Viala, Y. P., \& Bel, N. 1978, A\&A, 65, 435

D’Alessio, P., Cantó, J., Calvet, N., \& Lizano, S. 1998, ApJ, 500,411

Dent, W. R. F., Greaves, J. S., Mannings, V., Coulson, I. M., \& Walther, D. M. 1995, MNRAS, 277, L25

Draine, B. T. 1978, ApJS, 36, 595

Draine, B. T., \& Bertoldi, F. 1999, in The Universe as seen by ISO, ed. P. Cox, \& M. F. Kessler, ESA SP-427, 553

Dutrey, A., Guilloteau, S., Duvert, G., et al. 1996, A\&A, 309, 493

Feuerbacher, B., \& Fitton, B. 1972, J. Appl. Phys., 43, 1563

Gilman, R. C. 1972, ApJ, 178, 423

Habing, H. 1968, Bull. Astr. Inst. Netherlands, 19, 421
Hayashi, C., Nakazawa, K., \& Nakagawa, Y. 1985, in Protostars \& Planets II, ed. D. C. Black, \& M. S. Mathews (University of Arizona Press), 1100

Hollenbach, D. J., \& McKee, C. F. 1979, ApJS, 41, 555

Hollenbach, D. J., \& McKee, C. F. 1989, ApJ, 342, 306

Holweger, H., \& Rentzsch-Holm, I. 1995, A\&A, 303, 819

Holweger, H., Hempel, M., \& Kamp, I. 1999, A\&A, 350, 603

Jaquet, R., Staemmler, V., Smith, M. D., \& Flower, D. R. 1992, J. Phys. B, 25, 285

Jolly, A., McPhate, J. B., Lecavelier, A., et al. 1998, A\&A, 329, 1028

Kamp, I., \& Bertoldi, F. 2000, A\&A, 353, 276

Kamp, I., \& van Zadelhoff, G.-J. 2000, to appear in Planetary Systems in the Universe: Observation, Formation and Evolution, 7-10 August, ed. A. Penny, P. Artymowicz, A.M. Lagrange, \& S. Russell (Manchester, UK), Proc. of the IAU Symp. 202

Klahr, H., \& Lin, D. N. C. 2001, ApJ, in press

Kurucz, R. L. 1992, Rev. Mex. Astron. Astrofis., 23, 181

Launay, J. M., \& Roueff, E. 1977, A\&A, 56, 289

Le Bourlot, J., Pineau des Forêts, G., \& Flower, D. R. 1999, MNRAS, 305, 802

Liseau, R. 1999, A\&A, 348, 133

Mc Kee, C. F., Storey, J. W. V., Watson, D. M., \& Green, S. 1982, ApJ, 259, 647

Pantin, E., Lagage, P. O., \& Artymowicz, P. 1997, A\&A, 327, 1123

Press, W. H., Teukolsky, S. A., Vetterling, W. T., \& Flannery, B. P. 1997, Numerical Recipes in Fortran 77 (Cambridge University Press), 351

Roberge, A., Feldman, P. D., Lagrange, A. M., et al. 2000, ApJ, 538, 904

Sandford, S. A., \& Allamandola, L. J. 1990, Icarus, 87, 188

Schinke, R., Engel, V., Buck, U., Meyer, H., \& Diercksen, G. H. F. 1985, ApJ, 299, 939

Stephens, T. L., \& Dalgarno, A. 1973, ApJ, 186, 165

Sternberg, A., \& Dalgarno, A. 1989, ApJ, 338, 197

Telesco, C. M., \& Knacke, R. F. 1991, ApJ, 372, L29

Thi, W. F., Blake, G. A., van Dishoeck, E. F., et al. 2001, Nature, 409, 60

Tielens, A. G. G. M. 1983, ApJ, 271, 702

Tielens, A. G. G. M., \& Hollenbach, D. J. 1985, ApJ, 291, 722 van der Bliek, N. S., Prusti, T., \& Waters, L. B. F. M. 1994 A\&A, 285, 229

Watson, W. D. 1972, ApJ, 176, 103

Welsh, B. Y., Craig, N., Crawford, I. A., \& Price, R. J. 1998, A\&A, 338, 674

Yamashita, T., Handa, T., Omodaka, T., et al. 1993, ApJ, 402, L65 